\title{
The Impact of Rate Adaptation on Capacity-Delay Tradeoffs in Mobile Ad Hoc Networks
}

\author{
Cheng Wang, Member, IEEE, Xiang-Yang Li, Senior Member, IEEE, Changjun Jiang, Huiya Yan
}

\begin{abstract}
In this paper, we focus on the asymptotic capacity and delay, and their tradeoffs in mobile ad hoc networks (MANETs). As we all know, some fixed rate communication models such as the protocol model and the physical model have been studied in the past. However, our work aims to investigate the impact of an adaptive rate communication model on capacity-delay tradeoffs in MANETs under classical mobility models. Specifically, we adopt a well-known adaptive rate model called the generalized physical model (GphyM). The mobility of nodes is characterized by two broad classes of practical mobility models and they are hybrid random walk models and discrete random direction models. The two models generalize many mobility models studied in the literature, including the random walk, i.i.d., Brownian, and random way point models. For each mobility model, we derive the optimal delay for the optimal persession unicast capacity (that of constant order $\Theta(1)$ ) under the generalized physical model, depending on the individual parameters of mobility models. In particular, we show that for the i.i.d. model, compared with those under the protocol and physical models, the adaptive feature of link rate under the generalized physical model results in a significant decrease in the optimal delay for the optimal capacity; more precisely, both the optimal capacity and optimal delay can be simultaneously achieved, while there is no improvement for the random way-point model.
\end{abstract}

Index Terms-Capacity-Delay Tradeoffs; Mobile Ad Hoc Networks; Generalized Physical Model; Rate Adaptation

\section{INTRODUCTION}

The issue of capacity scaling laws for large scale wireless networks was pioneered by Gupta and Kumar [1]. Depending on the physical layer assumptions, the study of capacity scaling laws can be generally divided into two level$\mathrm{s}$ : information-theoretic level and networking-theoretic level. The information-theoretic bounds are usually addressed from an information-theoretic point of view, that is, without any particular assumption made on the way that communications take place [2]. Such studies fall into the scope of network information theory, and mainly characterize the fundamental limits of performance in multi-user communication problems. Those problems usually don't take account of the constraints imposed by state-of-the-art technology [3]. The networkingtheoretic bounds are usually derived under the assumption that the physical layer is restricted to perform simple point-topoint encoding and decoding, and the signals received from nodes other than one particular transmitter/sender are simply regarded as noise degrading the communication link [1], [4]. Such studies usually concentrate on the networking challenges involved. Therefore, the networking-theoretic capacity bounds

- Wang, Jiang and Li are with the Department of Computer Science and Engineering, Tongji University, and with the Key Laboratory of Embedded System and Service Computing, Ministry of Education, China. (E-mail: 3chengwang@gmail.com,cjjiang@online.sh.cn,xli@cs.iit.edu)

- Li is also with the Department of Computer Science and Technology, and TNLIST Tsinghua University, and the Department of Computer Science, Illinois Institute of Technology.

- Yan is with the Department of Mathematics, University of Wisconsin-La Crosse.(E-mail: hyan@uwlax.edu) are usually not larger than the information-theoretic ones for the same network deployment. Most studies investigate the capacities for different types of traffic, such as unicast, broadcast, multicast, anycast, many-to-one session, etc., under the frame of these two levels. In this paper, we study the networking-theoretic capacity and delay for unicast traffic in mobile ad hoc networks (MANETs).

In MANETs, the frequent changes of the topology due to the motion of nodes usually have a negative impact on system performance metrics, such as network delay [5] and data delivery ratio [6]. And those changes also bring fundamental challenges to both protocol design and performance analysis [7]. On the other hand, it was proved if the mobility is properly exploited, the node mobility can also improve certain performance metrics of system, e.g., network capacity [8] and connectivity [9]. The milestone work done by Grossglauser and Tse [8] showed that MANETs are scalable in terms of unicast capacity with assistance of mobility. However, the capacity in static ad hoc networks is not scalable without the help of advanced physical-layer techniques, e.g., cooperative MIMO communications [1], [4], [10]. In fact, such significant gain is obtained at the cost of a very large delay. Since capacity and delay are both paramount metrics in some applications of MANETs, it is necessary to examine relationships between them and they are the capacity-delay tradeoffs.

The delay and capacity in MANETs depend on the properties of the mobility models assumed. The mostly studied models include the i.i.d. model [11]-[13], the random walk model [14], the Brownian model [14]-[16], and the random waypoint model [15], [17]. Focusing on these models, extensive 
research has been devoted to improving capacity-delay tradeoffs for the data transport in MANETs by diverse techniques, such as packet redundancy scheme [11] and physical-layer or network-layer cooperative schemes [18].

In the literature, almost all relay policies are designed based on the fixed-rate communication model. In this scenario, if the value of a given conditional expression is beyond the threshold, the transmitter can send data successfully to the receiver at a specific constant data rate; otherwise, it cannot send data at any rate, i.e., the transmission rate is assumed to be a binary function. Both the protocol model and the physical model defined in [1] belong to this type of models [19].

In this paper, we aim to investigate the impact of the adaption of link rate on the delay-throughput tradeoffs in MANETs under the classical mobility models. Specifically, we adopt the generalized physical model [10], [20], a typical adaptiverate communication model, under which a link, say $v_{i} \rightarrow v_{j}$, achieves a continuous rate $R\left(v_{i}, v_{j}\right)=B \log \left(1+\operatorname{SINR}\left(v_{j}\right)\right)$, where $B$ is the channel bandwidth and $\operatorname{SINR}\left(v_{j}\right)$ is the signal to interference plus noise ratio at receiver $v_{j}$, see [21], [22]. Regarding mobility, we consider two broad classes of mobility models proposed in [17], called the hybrid random walk model (HRWMM) and the discrete random direction model (DRDMM) that involve a parameter called the degree of freedom. Other widely-used mobility models in the literature, such as the i.i.d., random walk, random way-point, and Brownian mobility models, can be regarded as the special cases of these two classes [17], [23] in terms of the degrees of freedom of nodes, denoted by $\gamma \in[0,1]$ for HRWMM and $\delta \in[0,1]$ for DRDMM, respectively.

Main Results: To the best of our knowledge, our work is the first one to study networking-theoretic scaling laws for MANETs under the adaptive-rate communication model. We focus on a random extended MANET, where $n$ mobile nodes are distributed randomly and uniformly on a square region $[0, \sqrt{n}] \times[0, \sqrt{n}]$ at the beginning. We mainly investigate the capacity and delay for MANETs under the well-known two-hop carry-and-forward communication paradigm without data replications [8] that has been extensively studied under the protocol and physical models. Those schemes follow a simple threshold-based principle: when the distance between two nodes is at most a threshold $d_{\mathbf{S}}$, these two nodes are requested to communicate directly; otherwise, they will communicate via the two-hop relay strategy. Depending on the critical parameter $d_{\mathbf{S}}:[1, \sqrt{n}]^{1}$ of a given two-hop strategy, say $\mathbf{S}$, under the generalized physical model with a power attenuation exponent $\alpha>2$, we derive the asymptotic persession capacity $\lambda(\mathbf{S}, n)$ and the corresponding average delay bounds $\mathbf{E}(D(\mathbf{S}, n))$ under both HRWMM and DRDMM. We highlight some selected interesting and insightful results as follows (The detailed summarization of results is provided in

1. For conciseness, we use $f(n):\left[\phi_{1}(n), \phi_{2}(n)\right]$ to represent that $f(n)=$ $\Omega\left(\phi_{1}(n)\right)$ and $f(n)=O\left(\phi_{2}(n)\right)$; let $f(n):\left(\phi_{1}(n), \phi_{2}(n)\right)$ represent that $f(n)=\omega\left(\phi_{1}(n)\right)$ and $f(n)=o\left(\phi_{2}(n)\right)$.
Section 4).

For HRWMM with the degree of freedom $\gamma \in[0,1]$ :

- We prove that under the generalized physical model, the feasible range of $d_{\mathbf{S}}$ to derive the optimal capacity, i.e., that of order $\Theta(1)$, for random extended MANETs is

$$
d_{\mathbf{S}}:\left[1, \min \left\{n^{\frac{\gamma}{2}} \sqrt{\log n}, \sqrt{n}\right\}\right],
$$

while under the protocol model and physical model the only feasible value of such $d_{\mathbf{S}}$ is $\Theta(1)$.

- For any $\gamma \in[0,1]$, under the premise of ensuring the optimal capacity, the network delay is nonincreasing with respect to the parameter $d_{\mathbf{S}}$; particularly, when $d_{\mathbf{S}}=\Theta(\sqrt{n})$, a transition of delay occurs. We indicate that this significant gain is obtained by the advantage of adaptive rate under the generalized physical model.

- For $\gamma=1$, i.e., the i.i.d. model, there is a transition for the optimal delay: the capacity and delay can be simultaneously achieved to the optimal order, i.e., $\Theta(1)$, by the simple two-hop strategy. It shows that this result breaks the limitations of capacity-delay tradeoffs derived under the protocol model or physical model in the literature, [24]-[31]. We point out that this "utopian" result comes from the extreme high freedom of nodes under the i.i.d. model $(\gamma=1)$ and the advantages of rate adaption; the transition is the result of the particularity of the i.i.d. model. More specifically, recall that under the i.i.d. mobility model the position of any node is independent of that in the adjacent time slots. This means that in the extended network the velocity of each node under the i.i.d. model (of order $\Theta(\sqrt{n})$ ) is increasing to infinity, as the network size $n \rightarrow \infty$. This feature of the i.i.d. model just contributes to this surprising improvement. To some extent, this result also gives a theoretical evidence that the i.i.d. model, a very special one, is not an appropriate model in analyzing the performance of practical mobile networks.

For DRDMM with the degree of freedom $\delta \in[0,1]$ :

- For the case of $0<\delta \leq 1$, which includes the random way-point model, it is necessary for achieving the optimal capacity of order $\Theta(1)$ to let $d_{\mathbf{S}}=\Theta(1)$.

- For the case of $\delta=0$, i.e., the discrete Brownian model, the optimal capacity is achievable when

$$
d_{\mathbf{S}}:[1, \sqrt{\log n}] .
$$

\section{For both HRWMM and DRDMM:}

- The capacity is independent of the power attenuation exponent of the generalized physical model, denoted by $\alpha$, as long as $\alpha>2$, while it decreases with $d_{\mathbf{S}}$ for some regimes. We indicate that the reason for this phenomenon lies in the fact that the data transmitted via long-distance links are indeed infinitesimal relative to those via short links when the link rate changes with the link length under the generalized physical model. The derivation can be found in the proofs of the main results: Theorem 1 and Theorem 2.

- The rate adaption can result in possible improvement of the capacity-delay tradeoffs for extended MANETs under 
some classical mobility models. Fig. 1 and Table. 1 show the comparison between the optimal delay corresponding to the optimal capacity for extended networks under the generalized physical model and that under the protocol model (or the physical model).

The rest of the paper is organized as follows. We formulate the system model in Section 2. In Section 3, we introduce the relay strategy, and propose the properties of mobile ad hoc extended networks, which will be used in the analysis of delay and capacity. In Section 4, we derive the main results, and propose some theoretical implications. In Section 5, we give an intuitive explanation of the impacts of rate adaptation and mobility models on the capacity-delay tradeoffs. Finally, we draw the conclusions and future perspectives in Section 6 .

\section{SySTEM MOdEL}

\subsection{Network Model}

In this paper, we focus on an ad hoc network with the random extended network model [4], [10], [19], [32], where $n$ mobile nodes are distributed randomly and uniformly on a square region $\mathcal{R}(n)=[0, \sqrt{n}] \times[0, \sqrt{n}]$ at time $t=0$. After that time, all mobile nodes move in accordance with the specific mobility model defined in Section 2.2. Assume there are $K$ unicast sessions, denoted by $\mathcal{K}(n, K)$, in the network. Each session, denoted by $k:\left\langle\mathcal{O}_{k}, \mathcal{D}_{k}\right\rangle \in \mathcal{K}(n, K)$, contains one source node $\mathcal{O}_{k}$ and the corresponding destination node $\mathcal{D}_{k}$. Each source node, say $\mathcal{O}_{k}$, generates traffic for its destination, say node $\mathcal{D}_{k}$, and the mapping $\mathcal{O}_{k} \mapsto \mathcal{D}_{k}$ is bijective. It is possible for a source node to send packets to its destination node via multiple relays. That is to say, a source node can send a packet to its destination node directly; or, the source node can transfer the packet to some relay nodes and let those relay nodes carry the packet to its destination. We focus on addressing unicast sessions in this work, and reserve for future work the analysis of other types of sessions, e.g., multicast and broadcast sessions.

\subsection{Random Mobility Model}

The i.i.d model, random walk model, random way-point model and Brownian model are widely studied classical models, but all of them can be regarded as special cases of HRWMM (hybrid random walk mobility model) or DRDMM (discrete random direction mobility model). So we study the two mobility models HRWMM and GRGMM in this paper.

We slightly modify the HRWMM and DRDMM proposed in [17] to make them applicable for the extended network.

Partition a square of area a into $\frac{\mathrm{a}}{\mathrm{c}}$ square cells of area c each, and let $\mathbb{L}(\mathbf{a}, \mathbf{c})$ denote the resulted lattice for the convenience of presentation. To avoid some trivialities, we assume that $\sqrt{\frac{\mathbf{a}}{\mathbf{c}}}$ is always an integer.

\subsubsection{Hybrid Random Walk Mobility Model}

We divide the deployment region $\mathcal{R}(n)=[0, \sqrt{n}]^{2}$ into $n$ squares of area 1 (henceforth referred to as cells) and that
TABLE 2

Notation used in this paper

\begin{tabular}{c|c}
\hline Notation & Meaning \\
\hline \hline $\mathbb{L}(\mathbf{a}, \mathbf{c})$ & lattice (partition a square of area a into cells of area $\mathbf{c})$ \\
\hline$L_{\mathrm{p}}^{\mathrm{h}}$ & duration of one phase under HRWMM \\
\hline$L_{\mathrm{p}}^{\mathrm{d}}$ & duration of one phase under DRDMM \\
\hline$L_{s}$ & duration of one static slot \\
\hline$L_{\mathrm{s}}^{t}$ & duration of one scheduling slot \\
\hline$i^{t}$ & link $i$ presented at static slot $t$ \\
\hline $\mathbf{t}_{i}^{t}$ and $\mathbf{r}_{i}^{t}$ & the transmitter and receiver of link $i$ at static slot $t$ \\
\hline $\mathcal{P}_{k, z}$ & a complete relay path for packet $z$ from session $k$ \\
\hline$i_{k, z}^{t}$ & the link used at slot $t_{i}$ for packet $z$ from session $k$ \\
\hline $\mathcal{S}^{t}$ & set of scheduled link at static slot $t$ \\
\hline$d_{\mathbf{S}}$ & critical distance \\
\hline
\end{tabular}

results in a lattice $\mathbb{L}(n, 1)$. We next divide the region $\mathcal{R}(n)$ into $n^{1-\gamma}$ squares of area $n^{\gamma}$ (henceforth referred to as super cells) and that results in a lattice $\mathbb{L}\left(n, n^{\gamma}\right)$, where $\gamma \in[0,1]$ is defined as the degree of freedom under the hybrid random walk mobility model. Clearly, there are $n^{\gamma}$ cells in each super cell. Each cell (or super cell) is located by the two-dimensional index $(x, y)$ if it is in the $(x+1)$ th column and $(y+1)$ th row of lattice $\mathbb{L}(n, 1)$ (or of lattice $\mathbb{L}\left(n, n^{\gamma}\right)$ ), in the order from the left to the right and from the bottom to the top, i.e., the cell in the bottom left corner has index $(0,0)$. To deal with the edge effects [1], we treat the deployment region $\mathcal{R}(n)$ as a twodimensional torus. The cells (or super cells) adjacent to cell (or super cell) $(i, j)$ are the cells (or super cells) $(i+1, j)$, $(i-1, j),(i, j+1)$, and $(i, j-1)$, where the addition and subtraction operations are performed modulo $n$ (or $n^{1-\gamma}$ ).

Time can be divided into phases of equal unit duration. Without loss of generality, we assume that the duration of each phase under the HRWMM is $L_{\mathrm{p}}^{\mathrm{h}}=1$. Initially, each node is equally likely to be in any of the cells and it is independent from the other nodes. At the beginning of each phase, a node uniformly chooses one cell at random from a randomly selected adjacent super cell, and jumps to the new cell from its current cell. Subsequently, the node will be presumed to be immobile in the new cell during this phase. An illustration is provided in Fig. 2(a).

In the special case $\gamma=1$, the HRWMM is essentially the i.i.d. mobility model (Fig. 2(b)); and for $\gamma=0$, it degenerates into the random walk model (Fig. 2(c)).

\subsubsection{Discrete Random Direction Mobility Model}

We divide the deployment region $\mathcal{R}(n)$ into a lattice $\mathbb{L}\left(n, n^{\delta}\right)$, where $\delta \in[0,1]$ is defined as the degree of freedom under the DRDMM. Moreover, time is divided into phases of equal duration $L_{\mathrm{p}}^{\mathrm{d}}=\Theta\left(n^{\frac{\delta}{2}}\right)$. Initially, each node is equally likely to be in any of the cells and it is independent from the other nodes. The motion of a node during this phase is as follows: at the beginning of each phase, a node uniformly chooses an end 


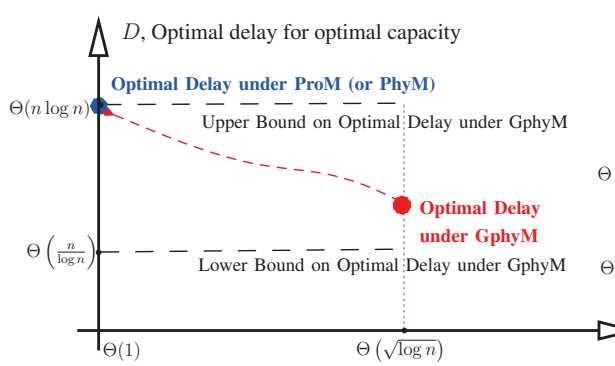

(a) $\gamma=0$ : Random Walk Model

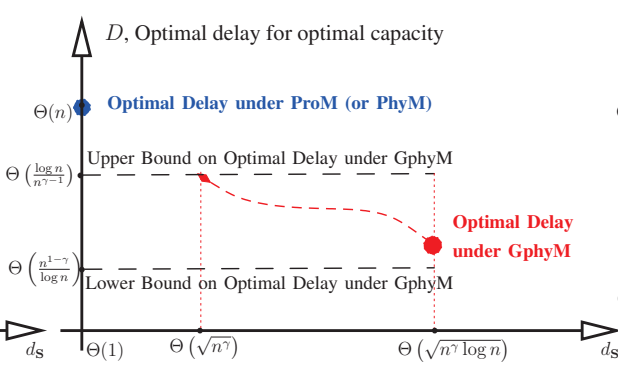

(b) $0<\gamma<1$ : General HRWMM

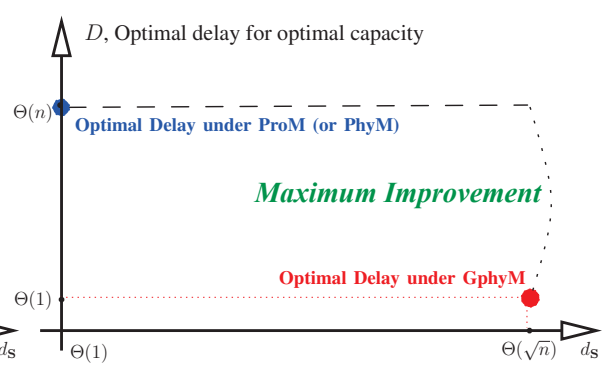

(c) $\gamma=1$ : I.I.D.Model

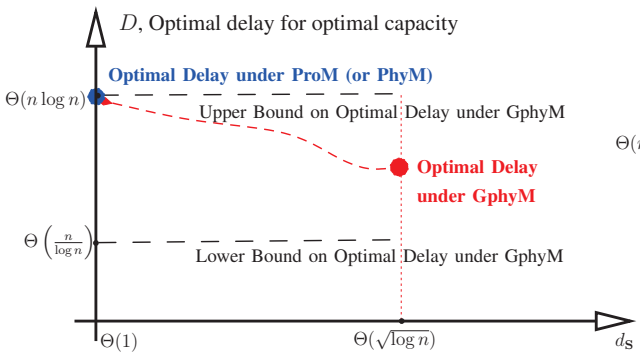

(d) $\delta=0$ : Brownian Mode

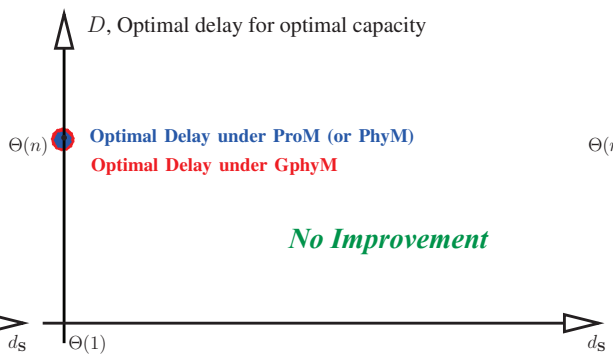

(e) $0<\delta<1$ : General DRDMM

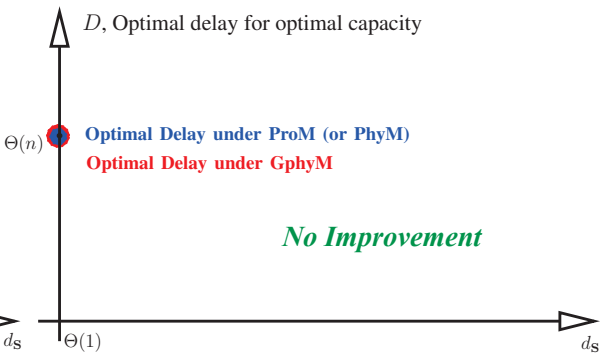

(f) $\delta=1$ : Random Way-Point Model

Fig. 1. Optimal delay for the optimal capacity (of order $\Theta(1)$ ) and the corresponding critical parameter $d_{\mathbf{S}}$ under the protocol model (ProM)/physical model (PhyM) and generalized physical model (GphyM).

TABLE 1

Optimal delay for the optimal capacity of order $(\Theta(1))$.

\begin{tabular}{r|c|c}
\hline & Delay under ProM (or PhyM) & Delay under GphyM \\
\hline \hline I.I.D Model, $\gamma=1$ & $D=\Theta(n)$ by $d_{\mathbf{S}}=\Theta(1)$ & $D=\Theta(1)$ by $d_{\mathbf{S}}=\Theta(\sqrt{n})$ \\
\hline Random Walk Model, $\gamma=0$ & $D=\Theta(n \log n)$ by $d_{\mathbf{S}}=\Theta(1)$ & $D=\Omega\left(\frac{n}{\log n}\right)$ by $d_{\mathbf{S}}:\left(1, \sqrt{\log n} ; ; \quad D=\Theta(n \log n)\right.$ by $d_{\mathbf{S}}=\Theta(1)$ \\
\hline General HRWMM, $0<\gamma<1$ & $D=\Theta(n)$ by $d_{\mathbf{S}}=\Theta(1)$ & $D=\Omega\left(\frac{n^{1-\gamma}}{\log n}\right)$ by $d_{\mathbf{S}}:\left(n^{\frac{\gamma}{2}}, n^{\frac{\gamma}{2}} \sqrt{\log n}\right] ; D=\Theta\left(\frac{\log n}{n^{\gamma-1}}\right)$ by $d_{\mathbf{S}}=\Theta\left(n^{\frac{\gamma}{2}}\right)$ \\
\hline Random Way-Point Model, $\delta=1$ & $D=\Theta(n)$ by $d_{\mathbf{S}}=\Theta(1)$ & $D=\Theta(n)$ by $d_{\mathbf{S}}=\Theta(1)$ \\
\hline Brownian Model, $\delta=0$ & $D=\Theta(n \log n)$ by $d_{\mathbf{S}}=\Theta(1)$ & $D=\Omega\left(\frac{n}{\log n}\right)$ by $d_{\mathbf{S}}:\left(1, \sqrt{\log n} ; \quad D=\Theta(n \log n)\right.$ by $d_{\mathbf{S}}=\Theta(1)$ \\
\hline General DRDMM, $0<\delta<1$ & $D=\Theta(n)$ by $d_{\mathbf{S}}=\Theta(1)$ & $D=\Theta(n)$ by $d_{\mathbf{S}}=\Theta(1)$ \\
\hline
\end{tabular}

point at random within a randomly selected adjacent cell, and moves to the end point at a velocity of constant order $\Theta(1)$, as in [17]. To keep the duration of all phases the same, the speed of the node is set in proportion to the distance between the start point and the end point.

In the special case $\delta=1$, the DRDMM is essentially similar to the random way-point mobility model (Fig. 2(e)); and for $\delta=0$, it degenerates into the discrete time version of the Brownian motion model (Fig. 2(f)).

\subsection{Communication Model}

When time is divided into slots of sufficiently small duration, it is reasonable to view each node as (approximately) in static status during a slot. We call such time slots static slots. Under the HRWMM, since the motion of every node happens instantaneously at the beginning of each phase, it follows that the position of each node remains invariable during a whole phase. Hence, for the HRWMM, we can set the duration of static slots to be $L_{\mathrm{s}}^{\mathrm{h}}:=L_{\mathrm{p}}^{\mathrm{h}}=1$. Under the DRDMM, each node moves with a velocity of constant order, which is infinitesimal relative to the extended scaling during a phase. Thus, it is acceptable to set the duration of static slots, denoted by $L_{\mathrm{s}}^{\mathrm{d}}$, to be a constant number. Without loss of generality, for the DRDMM, we also set $L_{\mathrm{s}}^{\mathrm{d}}=1$. Hence, we use $L_{\mathrm{s}}=1$ to denote the common duration of static slots under the HRWMM and DRDMM.

We remark that among $n$ mobile nodes, there are $n(n-1)$ possible directed communication links and each of them is associated with a unique transmitter-receiver pair. For any directed link $i$, we use $\mathbf{t}_{i}$ and $\mathbf{r}_{i}$ to denote its transmitter and receiver, respectively. Different from static networks, the position of each node may vary significantly as time goes on. For convenience, let $i^{t}, \mathbf{t}_{i}^{t}$ and $\mathbf{r}_{i}^{t}$ denote $i, \mathbf{t}_{i}$ and $\mathbf{r}_{i}$ that are presented during a static slot $t$, respectively. 

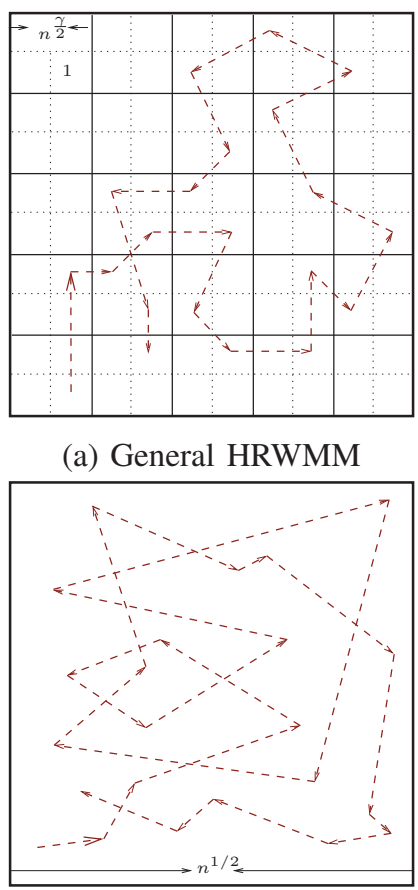

(b) I.I.D MM

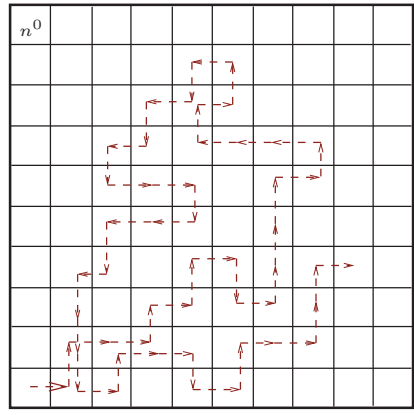

(c) RWMM (a) General HRWMM

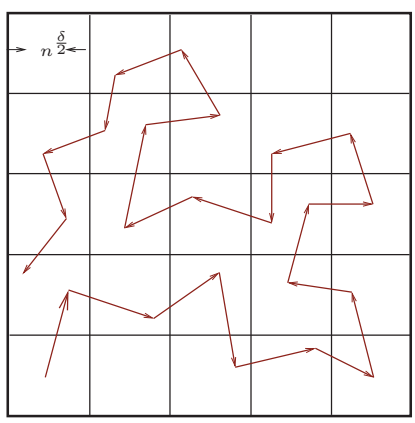

(d) General DRDMM

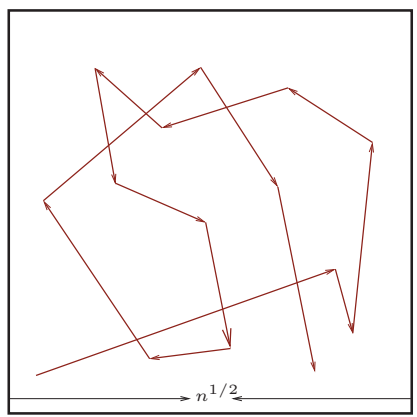

(e) RWPMM

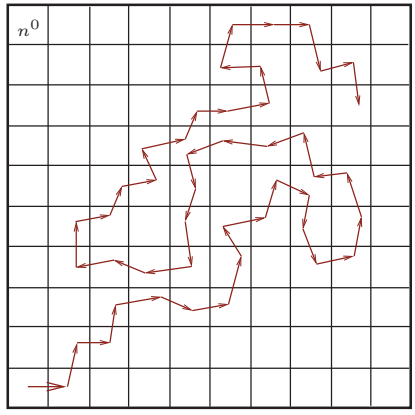

(f) $\mathrm{DBMM}$
Fig. 2. Illustrations of mobility models. (a)-(c) The motion of a node under the HRWMM. Each directed dashed segment along the motion track denotes a hop (jump) happening instantaneously at the beginning of a phase. (d)-(f) The motion of a node under the DRDMM. Each directed line segment along the motion track denotes a trip completed by the node throughout a whole phase. In order to keep the duration of all phases the same, the speed is set in proportion to the distance of every trip.

To address the feature of rate adaption, we adopt the generalized physical model (GphyM) [10], [32]. Assume that the bandwidth is $B=\Theta(1)$, and let $\|\cdot\|$ denote the Euclidean distance between two nodes.

Definition 1: Under the GphyM, for any scheduled set of links, say $\mathcal{S}^{t}$, the rate of a link, say $i^{t}$, is

$$
R_{i}^{\text {gau }, t}=B \times \mathbf{1} \cdot\left\{i^{t} \in \mathcal{S}^{t}\right\} \times \log \left(1+\operatorname{SINR}_{i}^{t}\right),
$$

where $\operatorname{SINR}_{i}^{t}=\frac{P \cdot \ell\left(\mathbf{t}_{i}^{t}, \mathbf{r}_{i}^{t}\right)}{N_{0}+\sum_{j \in \mathcal{S}^{t}-\{i\}} P \cdot \ell\left(\mathbf{t}_{j}^{t}, \mathbf{r}_{i}^{t}\right)}$, and $\ell(\cdot)$ is the power attenuation function.
Under the GphyM, we assume that the channel gain depends only on the distance between the transmitter and receiver, and ignore other fading effects, such as shadowing, [1], [10], [32]. For extended networks studied here, we follow the setting in [4], [10] and let $\ell\left(\mathbf{t}_{i}^{t}, \mathbf{r}_{i}^{t}\right)=\min \left\{1,\left\|\mathbf{t}_{i}^{t}-\mathbf{r}_{i}^{t}\right\|^{-\alpha}\right\}$, where $\alpha>2$ is the power attenuation exponent.

\subsection{Capacity and Delay}

We briefly introduce the concepts of capacity and delay in mobile ad hoc networks. Please refer to [8], [17], [33] for the detailed definitions.

\subsubsection{Network Capacity}

The network capacity is defined based on the stability of network. Data packets are assumed to arrive at node $v_{i}$ with probability $\lambda_{i}$ during each slot, i.e., in a Bernoulli process of arrival rate $\lambda_{i}$. The network is stable if there exists a scheduling scheme under which the queue at each node does not expand to infinity as time goes to infinity. Thus, the persession capacity of the network is the maximum rate that the network can stably support.

\subsubsection{Network Delay}

The delay for a packet is defined as the time it takes the packet to reach its destination after it arrives at the source. The total network delay is the expectation of the average delay over all packets in the long term.

\section{Communication Strategy}

Under a communication strategy, say $\mathbf{S}$, a key parameter is the so-called critical distance, denoted by $d_{\mathbf{S}}$, within which two nodes communicate directly with each other. It follows that $d_{\mathbf{S}}:[1, \sqrt{n}]$. For a specific mobility model, depending on a critical distance $d_{\mathbf{S}}$, we can define the contact interval during which data can be transmitted continually between the nodes with a distance of order $O\left(d_{\mathbf{S}}\right)$; and we can define the waiting interval as it takes a packet to wait for the next transmission at a relay node. We design the communication strategies in this paper based on the lattice $\mathbb{L}(\mathbf{a}, \mathbf{c})$ defined in Section 2.2. Then, under the strategy $\mathbf{S}$, we always insist that two nodes can communicate directly if they are located in the same cell in $\mathbb{L}\left(n,\left(d_{\mathbf{S}}\right)^{2}\right)$.

Under any communication strategy $\mathbf{S}$, a successful transmission (from the source to destination) of packet $z$ for session $k$ involves with a set of available links $\mathcal{R}_{k, z}$ that contains a complete relay path, denoted by

$$
\mathcal{P}_{k, z}=\left\{1_{k, z}^{t_{1}}, 2_{k, z}^{t_{2}}, \ldots, d_{k, z}^{t_{d}}\right\} \subseteq \mathcal{R}_{k, z} .
$$

To the best of our knowledge, this is the first work to study the capacity and delay scaling laws for MANETs under the adaptive-rate communication model. To concentrate on providing new insights of the impact of rate adaptation, we consider simple communication strategies without using the replication 


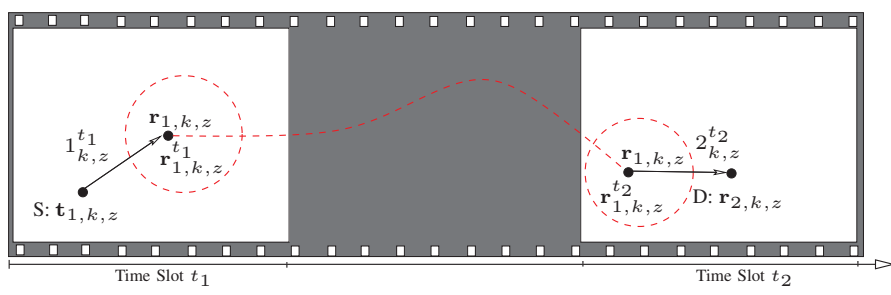

Fig. 3. Illustration of the routing path of packet $z$ for session $k, \mathcal{P}_{k, z}=\left\{1_{k, z}^{t_{1}}, 2_{k, z}^{t_{2}}\right\} \cdot \mathbf{t}_{1, k, z}$ is the source node, $\mathbf{r}_{2, k, z}$ is the destination node; $\mathbf{r}_{1, k, z}$ is the receiver of link $1_{k, z}^{t_{1}}$ and the transmitter of link $2_{k, z}^{t_{2}} \cdot \mathbf{r}_{1, k, z}^{t_{1}}$ and $\mathbf{r}_{1, k, z}^{t_{2}}$ denote the positions of node $\mathbf{r}_{1, k, z}$ in the static slot $t_{1}$ and $t_{2}$, respectively.

policy $^{2}$ [11] and reserve for future work the analysis of other advanced techniques, such as packet redundancy scheme [11] and physical-layer or network-layer cooperative schemes [18]. Hence, it holds that $\mathcal{R}_{k, z}=\mathcal{P}_{k, z}$ in this work.

\subsection{Two-Hop Relay Strategy}

The two-hop strategy was first proposed by Grossglauser and Tse [8]. Under the two-hop strategy, for each packet $z$ for session $k$, the complete relay path can be denoted by $\mathcal{P}_{k, z}=$ $\left\{1_{k, z}^{t_{1}}, 2_{k, z}^{t_{2}}\right\}$. As illustrated in Fig. 3, there are generally three phases under the two-hop strategy: (1) $\mathrm{S} \rightarrow \mathrm{R}$ phase, during which the source node $\mathbf{t}_{1, k, z}$ transmits the packet $z$ to a relay node $\mathbf{r}_{1, k, z}$, i.e., $\mathbf{t}_{2, k, z}$; (2) waiting phase, during which $\mathbf{r}_{1, k, z}$ holds the packet $z$ until it meets the destination node $\mathbf{r}_{2, k, z}$ within a distance of $d_{\mathbf{S}}$, and (3) $\mathrm{R} \rightarrow \mathrm{D}$ phase, during which $\mathbf{r}_{1, k, z}$ transmits the packet $z$ to $\mathbf{r}_{2, k, z}$.

Note that the durations of $S \rightarrow R$ and $R \rightarrow D$ phases are of the same order as the contact time with the parameter $d_{\mathbf{S}}$ (Definition B.3 in Appendix B); the duration of the waiting phase can be derived based on the first hitting time (Definition B.1, Corollary B.1 in Appendix B). The $\mathrm{S} \rightarrow \mathrm{R}$ and $\mathrm{R} \rightarrow \mathrm{D}$ phases are the contact intervals, while the waiting phase is the waiting interval. We provide an illustration in Fig. 4, and will determine those durations in Sections 3.2.2 and 3.2.3.

\subsection{Properties of Mobile Networks}

The following to be examined properties of networks depend on the value of $d_{\mathbf{S}}$ that denotes the critical distance of a given communication strategy $\mathbf{S}$.

\subsubsection{Spatial Multiplexing in Extended MANETs}

Intuitively, there is a tradeoff between the network throughput and delay according to the critical distance $d_{\mathbf{S}}$. We carry out our analysis based on lattice $\mathbb{L}(n, \mathbf{c}(n))$, where $\mathbf{c}(n)>2$. Denote the cell in the $(i+1)$ th row and $j+1$ th column by $C_{i, j}$, and denote the number of mobile nodes in $C_{i, j}$ as $n_{i, j}$.

2. Under the replication policy, the source node can send duplicate copies of the packet to new relay nodes.

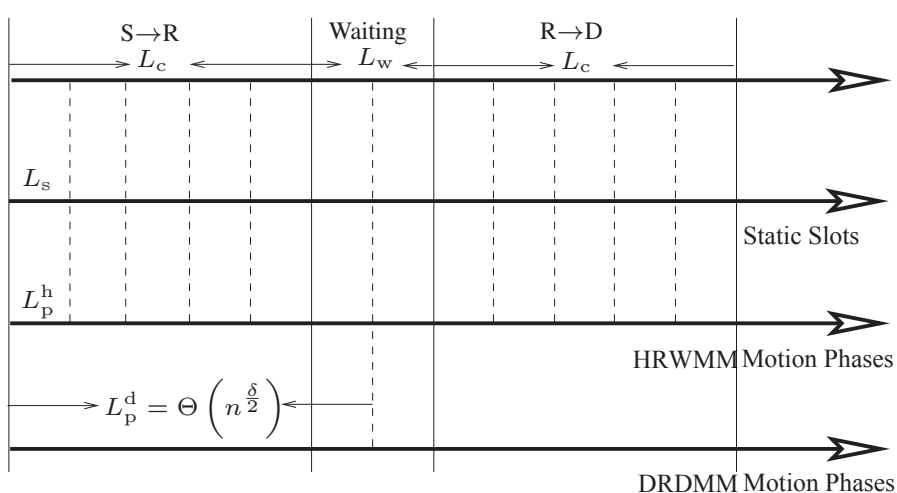

Fig. 4. Decomposition of two-hop communication. $L_{\mathrm{c}}$ and $L_{\mathrm{w}}$ are the average durations of the contact intervals and waiting intervals, respectively. $L_{\mathrm{s}}$ is the duration of static slots. $L_{\mathrm{p}}^{\mathrm{h}}$ and $L_{\mathrm{p}}^{\mathrm{d}}$ are the durations of phases under the HRWMM and DRDMM, respectively; $L_{\mathrm{p}}^{\mathrm{h}}=L_{\mathrm{s}}=1$ and $L_{\mathrm{p}}^{\mathrm{d}}=\Theta\left(n^{\frac{\delta}{2}}\right), 0 \leq \delta \leq 1$. Note that the contact intervals and waiting intervals can always be divided into static slots; under the HRWMM, they can also be divided into motion phases; while, under the DRDMM, they are not necessarily divided into motion phases due to the continuous motion of nodes.

Furthermore, we define a sequence of sets of cells. For $h=$ $0,1, \ldots, \mu, v=0,1, \ldots, \mu$, and $0 \leq i, j \leq \sqrt{\frac{n}{\mathbf{c}(n)}}-1$, we define a set of cells as

$$
\mathcal{C}_{h, v}(\mu):=\left\{C_{i, j} \mid i \bmod (\mu+1)=h, j \bmod (\mu+1)=v\right\},
$$

where $\mu \geq 1$ is an integer. Then,

$$
\left|\mathcal{C}_{h, v}(\mu)\right|=\frac{1}{(\mu+1)^{2}} \cdot \frac{n}{\mathbf{c}(n)},
$$

where $|\cdot|$ denotes the cardinality of a discrete set.

Lemma 1: Under any stationary and ergodic mobility model, at any time $t$, the following holds:

1) When $\mathbf{c}(n)=O(\log n)$, define the number of cells in $\mathcal{C}_{h, v}(\mu)$ that contain at least 2 nodes as a random variable $\xi$, then there is a constant $\theta_{1}>0$ with high probability such that

$$
\xi \geq \theta_{1} \cdot \frac{n}{\mathbf{c}(n)} .
$$

2) When $\mathbf{c}(n)=\Omega(\log n)$, for all cells in lattice $\mathbb{L}(n, \mathbf{c}(n))$, the number of nodes is uniformly of order $\Theta(\mathbf{c}(n))$ with high probability.

Proof: The first result can be easily proven by Chebychev's inequality (Lemma A.1 in Appendix A). The second result can be proven by a similar method to that of Lemma 18 in [34] based on VC Theorem in [35]. All proofs are based on the fact that under any stationary and ergodic mobility model, the distribution of nodes at all times remains uniform. Due to the similarity, we omit this proof. 
Next, we present an important result of the spatial multiplexing in the extended MANETs of interest, i.e., Lemma 2. We give an illustration of Lemma 2 in Fig. 5.

Lemma 2: Under the generalized physical model (GphyM) with $\alpha>2$, when $d_{\mathbf{S}}=\Omega(1)$, there exists a strategy $\mathbf{S}$ under which there is a scheduling set $\mathcal{S}^{t}$ such that:

1) $\left|\mathcal{S}^{t}\right| \geq \kappa_{0} \cdot n$, with high probability, where $\left|\mathcal{S}^{t}\right|$ represents the total number of links contained in $\mathcal{S}^{t}$, and $\kappa_{0}>0$ is a constant;

2) for any link $i^{t} \in \mathcal{S}^{t}$, it holds that $R_{i}^{t}=\Omega\left(\left(d_{\mathbf{S}}\right)^{-\alpha}\right)$.

Proof: We prove this lemma according to different values of the critical distance $d_{\mathbf{S}}$.

(1) When $d_{\mathbf{S}}=\Theta(1)$, i.e., $\mathbf{c}(n)=\Theta(1)$

At a static slot $t$, for any $\mathcal{C}_{h, v}(\mu)$, according to the first result of Lemma 1 , there are at least $\theta_{1} \cdot \frac{n}{\mathbf{c}(n)}$ cells containing 2 nodes. Since all links are limited within the same cells, we can choose a link from each cell to construct the set $\mathcal{S}^{t}$. Obviously, it holds that $\left|\mathcal{S}^{t}\right| \geq \theta_{1} \cdot n / \mathbf{c}(n)$. For any link $i^{t} \in \mathcal{S}^{t}$, the strength of signal at the receiver $\mathbf{r}_{i}^{t}$ is

$$
\mathrm{S}_{i}^{t} \geq P \cdot \min \left\{1,(\sqrt{2 \cdot \mathbf{c}(n)})^{-\alpha}\right\}=\Theta(1)
$$

The total interference at $\mathbf{r}_{i}^{t}$ is bounded by

$$
\mathrm{I}_{i}^{t} \leq 8 P \cdot(\mathbf{c}(n))^{-\frac{\alpha}{2}} \cdot \sum_{k=0}^{\infty} \frac{1}{((\mu+1) \cdot k+\mu)^{\alpha}} .
$$

Thus, it follows that $\mathrm{I}_{i}^{t}=O(1)$ for $\alpha>2$. According to Equation (1), it holds that $R_{i}^{t}=\Theta(1)$. Furthermore, since $\mathbf{c}(n)=\Theta(1)$, we get that $R_{i}^{t}=\Omega\left((\mathbf{c}(n))^{-\frac{\alpha}{2}}\right)$.

(2) When $d_{\mathbf{S}}=\Omega(\sqrt{\log n})$, i.e., $\mathbf{c}(n)=\Omega(\log n)$

By the second result of Lemma 1, there is a constant $\theta_{3}>0$ such that $n_{i, j} \geq \theta_{3} \cdot \mathbf{c}(n)$ for all cells in $\mathbb{L}(n, \mathbf{c}(n))$. Next, we construct the set $\mathcal{S}^{t}$. From any cell $C_{i, j} \in \mathcal{C}_{h, v}(3)$, choose $\theta_{3} \cdot \mathbf{c}(n)$ nodes, and correspondingly choose $\theta_{3} \cdot \mathbf{c}(n)$ nodes from the cell $C_{i, j+2}$ (or $C_{i+2, j}$ ). We make these $\theta_{3} \cdot \mathbf{c}(n)$ pairs communicate directly to build $\theta_{3} \cdot \mathbf{c}(n)$ links, and finally obtain the set $\mathcal{S}^{t}$ that consists of $\left|\mathcal{C}_{h, v}(3)\right| \cdot 2 \theta_{3} \cdot \mathbf{c}(n)$ links. Then, $\left|\mathcal{S}^{t}\right| \geq \theta_{2} \cdot n$, where $\theta_{2} \leq \frac{1}{8} \cdot \theta_{3}$.

For any link $i^{t} \in \mathcal{S}^{t}$, the signal strength at $\mathbf{r}_{i}^{t}$ is

$$
\mathrm{S}_{i}^{t} \geq P \cdot(\sqrt{10 \cdot \mathbf{c}(n)})^{-\alpha}=\Omega\left((\mathbf{c}(n))^{-\frac{\alpha}{2}}\right) .
$$

The total interference at $\mathbf{r}_{i}^{t}$ is bounded by

$$
\begin{aligned}
\mathrm{I}_{i}^{t} \leq & P \cdot\left(\theta_{3} \cdot \mathbf{c}(n)-1\right) \cdot(\mathbf{c}(n))^{-\frac{\alpha}{2}} \\
& +8 P \cdot \theta_{3} \cdot \mathbf{c}(n) \cdot(\mathbf{c}(n))^{-\frac{\alpha}{2}} \sum_{k=0}^{\infty} \frac{1}{(4 k+1)^{\alpha}} \\
\leq & P \cdot\left(1+8 \theta_{4}\right) \cdot(\mathbf{c}(n))^{1-\frac{\alpha}{2}}
\end{aligned}
$$

where $\theta_{4}>\sum_{k=0}^{\infty} \frac{1}{(4 k+1)^{\alpha}}$ is a constant with $\alpha>2$, then $\mathrm{I}_{i}^{t}=O\left((\mathbf{c}(n))^{1-\frac{\alpha}{2}}\right)$. That is, $\mathrm{I}_{i}^{t}=o(1)$. According to (1), it holds that $R_{i}^{t}=\Omega\left((\mathbf{c}(n))^{-\frac{\alpha}{2}}\right)$.
(3) When $d_{\mathbf{S}}=\Omega(1)$ and $d_{\mathbf{S}}=O(\sqrt{\log n})$, i.e., $\mathbf{c}(n)=\Omega(1)$ and $\mathbf{c}(n)=O(\log n)$

By applying the first result of Lemma 1 and a similar procedure of the case when $d_{\mathbf{S}}=\Omega(\sqrt{\log n})$, we can prove the result of this case.

From Lemma 2, the following result obviously holds.

Lemma 3: During any static slot $t$, the total throughput of order $\Omega\left(n \cdot\left(d_{\mathbf{S}}\right)^{-\alpha}\right)$ is achievable with high probability under the strategy with a critical distance $d_{\mathbf{S}}$.

\subsubsection{Duration of Contact Intervals}

Now, we derive the duration of contact intervals in a given strategy $\mathbf{S}$ under the HRWMM and DRDMM, denoted by $\tau_{C}^{\mathrm{h}}\left(d_{\mathbf{S}}\right)$ and $\tau_{C}^{\mathrm{d}}\left(d_{\mathbf{S}}\right)$, respectively.

Lemma 4: Under the HRWMM and the strategy $\mathbf{S}$ with a critical distance $d_{\mathbf{S}}$, with high probability, $\mathbf{E}\left(\tau_{C}^{\mathrm{h}}\left(d_{\mathbf{S}}\right)\right)$ is of order

$$
\left\{\begin{array}{lll}
\Omega\left(\frac{\left(d_{\mathbf{S}}\right)^{2}}{\log n \cdot n^{\gamma}}\right) & \text { when } & d_{\mathbf{S}}:\left[n^{\frac{\gamma}{2}} \cdot \sqrt{\log n}, \sqrt{n}\right], \\
\Omega(1) & \text { when } & d_{\mathbf{S}}:\left[1, n^{\frac{\gamma}{2}} \cdot \sqrt{\log n}\right] .
\end{array}\right.
$$

Proof: Based on Lemma B.2 in Appendix B, we directly study the first exit time to derive the order of contact time. Let $\tau_{E}^{\mathrm{h}}\left(d_{\mathbf{S}}\right)$ denote the first exit time under the HRWMM.

Consider a node, say $i$, and denote the cells containing it at time 0 and $t$ by $\left(x_{0}, y_{0}\right)$ and $\left(x_{t}, y_{t}\right)$, respectively; denote its positions at time 0 and $t$ by $i^{0}$ and $i^{t}$, respectively.

(1) When $d_{\mathbf{S}}=\Omega\left(n^{\frac{\gamma}{2}} \cdot \sqrt{\log n}\right)$

Define two random variables as follows:

$$
\begin{aligned}
\tau_{E}^{\mathrm{h}, x}(h) & :=\inf \left\{t \geq 0:\left|x_{t}-x_{0}\right| \geq h\right\}, \\
\tau_{E}^{\mathrm{h}, y}(v) & :=\inf \left\{t \geq 0:\left|y_{t}-y_{0}\right| \geq v\right\} .
\end{aligned}
$$

By a geometric argument (Pythagoras' Theorem), we have that if $\left\|i^{t}-i^{0}\right\| \geq d_{\mathbf{S}}$ holds, then at least one of $\left|x_{t}-x_{0}\right|$ and $\left|y_{t}-y_{0}\right|$ is not less than $\left\lfloor\frac{d_{\mathbf{S}}}{\sqrt{2} \cdot n^{\frac{\gamma}{2}}}\right\rfloor$. Combining Definition B.2 in Appendix B and the fact that

$$
\frac{d_{\mathbf{S}}}{2 n^{\frac{\gamma}{2}}} \leq\left\lfloor\frac{d_{\mathbf{S}}}{\sqrt{2} \cdot n^{\frac{\gamma}{2}}}\right\rfloor
$$

we get that for any integer $L \geq 0$, if $\tau_{E}^{\mathrm{h}}\left(d_{\mathbf{S}}\right) \leq L$, then

$$
\tau_{E}^{\mathrm{h}, x}\left(\frac{d_{\mathbf{S}}}{2 n^{\frac{\gamma}{2}}}\right) \leq L \quad \text { or } \quad \tau_{E}^{\mathrm{h}, y}\left(\frac{d_{\mathbf{S}}}{2 n^{\frac{\gamma}{2}}}\right) \leq L,
$$

where we assume that $\frac{d_{\mathbf{S}}}{2 n^{\frac{\gamma}{2}}}$ is always an integer to simplify the description. Therefore, it follows that

$\mathbf{P}\left(\tau_{E}^{\mathrm{h}}\left(d_{\mathbf{S}}\right) \leq L\right) \leq \mathbf{P}\left(\tau_{E}^{\mathrm{h}, x}\left(\frac{d_{\mathbf{S}}}{2 n^{\frac{\gamma}{2}}}\right) \leq L \vee \tau_{E}^{\mathrm{h}, y}\left(\frac{d_{\mathbf{S}}}{2 n^{\frac{\gamma}{2}}}\right) \leq L\right)$.

Using union bounds and the symmetry of node motion, we can obtain that

$$
\mathbf{P}\left(\tau_{E}^{\mathrm{h}}\left(d_{\mathbf{S}}\right) \leq L\right) \leq 2 \cdot \mathbf{P}\left(\tau_{E}^{\mathrm{h}, x}\left(\frac{d_{\mathbf{S}}}{2 n^{\frac{\gamma}{2}}}\right) \leq L\right) .
$$

Before the phase $\tau_{E}^{\mathrm{h}}\left(d_{\mathbf{S}}\right)$, it holds that $x_{t}=x_{0}+\sum_{i=0}^{t} s_{i}$, where $s_{i}, i=1,2, \ldots, t$, are i.i.d. random variables taking 


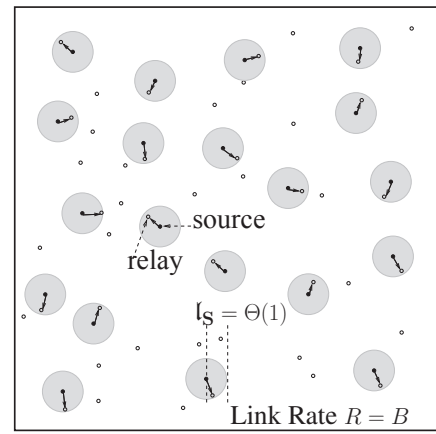

(a) Phase 1 under Fixed-Rate Model

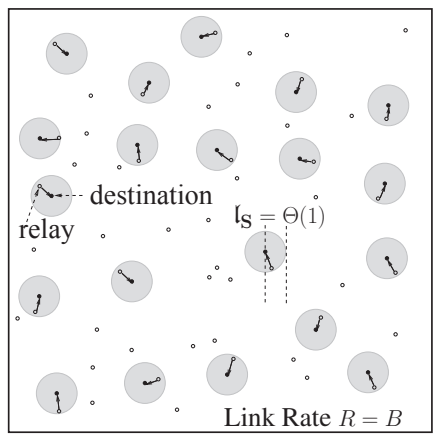

(b) Phase 2 under Fixed-Rate Model

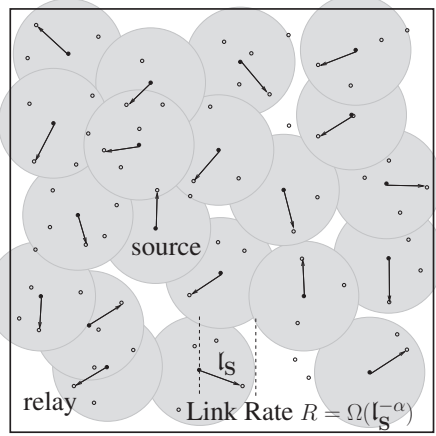

(c) Phase 1 under GphyM

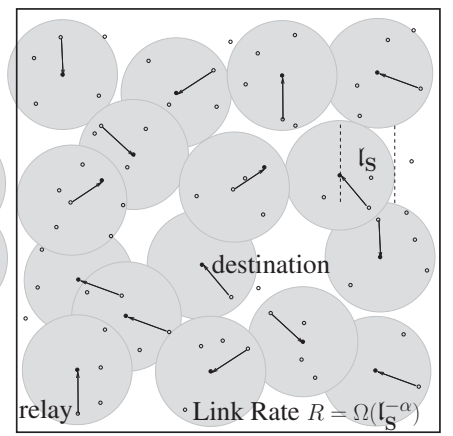

(d) Phase 2 under GphyM

Fig. 5. Under the fixed-rate model (F-R model, Figures (a) and (b)), e.g., the protocol model and physical model, the only feasible setting of $d_{\mathbf{S}}$ for achieving the optimal capacity of $\Theta(1)$ for random extended networks is of $d_{\mathbf{S}}=\Theta(1)$, which potentially causes large delays. During each time slot, there are $\Theta(n)$ feasible links (sender-receiver pairs) of length $O(1)$ along which a fixed constant rate can be sustained. Under the generalized physical model (GphyM, Figures (c) and (d)), the link rate is adjustable according to SINR at the receiver. It is possible to achieve a constant capacity with $d_{\mathbf{S}}=\omega(1)$, which possibly decreases the network delay. In each slot, $\Theta(n)$ links of length $O\left(d_{\mathbf{S}}\right)$ can simultaneously achieve the rate of order $\Omega\left(d_{\mathbf{S}}^{-\alpha}\right)$, where $\alpha>2$ is the power attenuation exponent.

values in $\{-1,0,1\}$ with probabilities $\left\{\frac{1}{4}, \frac{1}{2}, \frac{1}{4}\right\}$, respectively. Due to the symmetry of $x_{t}$, it is clear that the reflection principle for 1-D random walk also holds in case of $x_{t}$. Thus, we have

$$
\mathbf{P}\left(\tau_{E}^{\mathrm{h}, x}\left(\frac{d_{\mathbf{S}}}{2 n^{\frac{\gamma}{2}}}\right) \leq L\right) \leq 2 \cdot \mathbf{P}\left(x_{\lfloor L\rfloor}-x_{0} \geq \frac{d_{\mathbf{S}}}{2 n^{\frac{\gamma}{2}}}\right),
$$

where $\lfloor\cdot\rfloor$ denotes the greatest integer function. Since each $s_{i}$ has mean 0 and variance $\frac{1}{2}, \operatorname{Var}\left(x_{t}-x_{0}\right)=\frac{t}{2}$. By using Lemma A.2 in Appendix A, we have, for $t \geq \frac{d_{\mathrm{S}}^{\frac{\gamma}{2}}}{2 n^{\frac{\gamma}{2}}}$,

$$
\begin{aligned}
\mathbf{P}\left(x_{t}-x_{0} \geq \frac{d_{\mathbf{S}}}{2 n^{\frac{\gamma}{2}}}\right) & =\mathbf{P}\left(x_{t}-x_{0} \geq \sqrt{\frac{t}{2}} \cdot \frac{d_{\mathbf{S}}}{\sqrt{2 t} \cdot n^{\frac{\gamma}{2}}}\right) \\
& \leq \exp \left(-\left(d_{\mathbf{S}} / n^{\frac{\gamma}{2}}\right)^{2} \cdot \frac{1}{8 t}\right) .
\end{aligned}
$$

Let $L=\frac{\left(d_{\mathbf{S}}\right)^{2}}{16 \log n \cdot n^{\gamma}}$, we have

$$
\mathbf{P}\left(\tau_{E}^{\mathrm{h}}\left(d_{\mathbf{S}}\right) \leq \frac{\left(d_{\mathbf{S}}\right)^{2}}{16 \log n \cdot n^{\gamma}}\right) \leq \frac{4}{n^{2}} .
$$

Then, $\mathbf{E}\left(\tau_{E}^{\mathrm{h}}\left(d_{\mathbf{S}}\right)\right)=\Omega\left(\frac{\left(d_{\mathbf{S}}\right)^{2}}{\log n \cdot n^{\gamma}}\right)$.

(2) When $d_{\mathbf{S}}=O\left(n^{\frac{\gamma}{2}} \cdot \sqrt{\log n}\right)$

It is straightforward that $\tau_{E}^{\mathrm{h}}\left(d_{\mathbf{S}}\right) \geq L_{\mathrm{p}}^{\mathrm{h}}$. Since $L_{\mathrm{p}}^{\mathrm{h}}=\Theta(1)$, we have that $\mathbf{E}\left(\tau_{E}^{\mathrm{h}}\left(d_{\mathbf{S}}\right)\right)=\Omega(1)$.

Lemma 5: Under the DRDMM and the strategy $\mathbf{S}$ with a critical distance $d_{\mathbf{S}}$, with high probability, $\mathbf{E}\left(\tau_{C}^{\mathrm{d}}\left(d_{\mathbf{S}}\right)\right)$ is of order

$$
\left\{\begin{array}{lll}
\Omega\left(\frac{\left(d_{\mathbf{S}}\right)^{2}}{\log n \cdot n^{\frac{\delta}{2}}}\right) & \text { when } & d_{\mathbf{S}}:\left[n^{\frac{\delta}{2}} \cdot \sqrt{\log n}, \sqrt{n}\right], \\
\Omega\left(n^{\frac{\delta}{2}}\right) & \text { when } & d_{\mathbf{S}}:\left[n^{\frac{\delta}{2}}, n^{\frac{\delta}{2}} \sqrt{\log n}\right] \cap[1, \sqrt{n}, \\
\Omega\left(d_{\mathbf{S}}\right) & \text { when } & d_{\mathbf{S}}:\left[1, n^{\frac{\delta}{2}}\right] .
\end{array}\right.
$$

Proof: In a similar way of the proof of Lemma 4, we derive the order of contact time by computing the first exit time under the DRDMM denoted by $\tau_{E}^{\mathrm{d}}\left(d_{\mathbf{S}}\right)$.

(1) When $d_{\mathbf{S}}=\Omega\left(n^{\frac{\delta}{2}} \cdot \sqrt{\log n}\right)$

Without loss of generality, we consider a node $i$ that is located at the position $(0,0)$ at time 0 . Let $\left(x_{t}, y_{t}\right)$ denote the position of node $i$ after $t$ trips. Define

$$
\begin{aligned}
\tau_{E}^{\mathrm{d}, x}(h) & :=\inf \left\{t \geq 0:\left|x_{t}\right| \geq h\right\}, \\
\tau_{E}^{\mathrm{d}, y}(v) & :=\inf \left\{t \geq 0:\left|y_{t}\right| \geq v\right\} .
\end{aligned}
$$

Then, it holds that

$\mathbf{P}\left(\tau_{E}^{\mathrm{d}}\left(d_{\mathbf{S}}\right) \leq L\right) \leq \mathbf{P}\left(\tau_{E}^{\mathrm{d}, x}\left(\frac{d_{\mathbf{S}}}{\sqrt{2}}\right) \leq L \vee \tau_{E}^{\mathrm{d}, y}\left(\frac{d_{\mathbf{S}}}{\sqrt{2}}\right) \leq L\right)$.

By the union bounds and the symmetry of node motion, we get

$$
\mathbf{P}\left(\tau_{E}^{\mathrm{d}}\left(d_{\mathbf{S}}\right) \leq L\right) \leq 2 \cdot \mathbf{P}\left(\tau_{E}^{\mathrm{d}, x}\left(\frac{d_{\mathbf{S}}}{\sqrt{2}}\right) \leq L\right) .
$$

Define the $x$-coordinate of the position of a node after finishing the $k$ th trip. Then, before time $\tau_{E}^{\mathrm{d}}\left(d_{\mathbf{S}}\right)$, it holds for $s_{k}: s_{k}=$ $\sum_{i=1}^{k} z_{i}$, where $z_{i}, i=1,2, \ldots, k$, are i.i.d. random variables taking values in $\left[-n^{\frac{\delta}{2}}, n^{\frac{\delta}{2}}\right]$. Since

$$
\mathbf{E}\left(z_{i}\right)=0, \operatorname{Var}\left(z_{i}\right)=\frac{n^{\delta}}{3} \text {, and } \operatorname{Var}\left(s_{k}\right)=k \cdot \frac{n^{\delta}}{3},
$$

according to Lemma A.2, we have, for $k \geq \frac{\sqrt{6}}{2} \cdot \frac{d_{\mathrm{S}}}{n^{\frac{\delta}{2}}}$,

$$
\begin{aligned}
\mathbf{P}\left(s_{k} \geq \frac{d_{\mathbf{S}}}{\sqrt{2}}\right) & =\mathbf{P}\left(s_{k} \geq n^{\frac{\delta}{2}} \cdot \sqrt{\frac{k}{3}} \cdot \frac{\sqrt{3} \cdot d_{\mathbf{S}}}{n^{\frac{\delta}{2}} \cdot \sqrt{2 k}}\right) \\
& \leq \exp \left(-\left(d_{\mathbf{S}} / n^{\frac{\delta}{2}}\right)^{2} \cdot \frac{3}{8 k}\right) .
\end{aligned}
$$


By the symmetry of the node motion, we have

$$
\mathbf{P}\left(\left|s_{k}\right| \geq \frac{d_{\mathbf{S}}}{\sqrt{2}}\right) \leq 2 \exp \left(-\left(d_{\mathbf{S}} / n^{\frac{\delta}{2}}\right)^{2} \cdot \frac{3}{8 k}\right) .
$$

Recall that the duration of the phases is $L_{\mathrm{p}}^{\mathrm{d}}=\Theta\left(n^{\frac{\delta}{2}}\right)$, we assume that $L_{\mathrm{p}}^{\mathrm{d}}=\rho \cdot n^{\frac{\delta}{2}}$ with a constant $\rho>0$. Since

$$
\mathbf{P}\left(\tau_{E}^{\mathrm{d}, x}\left(\frac{d_{\mathbf{S}}}{\sqrt{2}}\right) \leq k \cdot \rho \cdot n^{\frac{\delta}{2}}\right)=\mathbf{P}\left(\bigcup_{i=1}^{k}\left|s_{i}\right| \geq \frac{d_{\mathbf{S}}}{\sqrt{2}}\right),
$$

it holds that

$$
\mathbf{P}\left(\tau_{E}^{\mathrm{d}, x}\left(\frac{d_{\mathbf{S}}}{\sqrt{2}}\right) \leq k \cdot \rho \cdot n^{\frac{\delta}{2}}\right) \leq 2 k \cdot \exp \left(-\left(\frac{d_{\mathbf{S}}}{n^{\frac{\delta}{2}}}\right)^{2} \cdot \frac{3}{8 k}\right) \text {. }
$$

Then, combining Equations (5) and (6), we obtain

$$
\mathbf{P}\left(\tau_{E}^{\mathrm{d}}\left(d_{\mathbf{S}}\right) \leq k \cdot \rho \cdot n^{\frac{\delta}{2}}\right) \leq 4 k \cdot \exp \left(-\left(\frac{d_{\mathbf{S}}}{n^{\frac{\delta}{2}}}\right)^{2} \cdot \frac{3}{8 k}\right) .
$$

Let $k=\frac{\left(d_{\mathbf{S}}\right)^{2}}{8 \log n \cdot n^{\delta}}$, we get

$$
\mathbf{P}\left(\tau_{E}^{\mathrm{d}}\left(d_{\mathbf{S}}\right) \leq \frac{\rho \cdot\left(d_{\mathbf{S}}\right)^{2}}{8 \log n \cdot n^{\frac{\delta}{2}}}\right) \leq \frac{4}{n^{2}} .
$$

Then, it holds that $\mathbf{E}\left(\tau_{E}^{\mathrm{d}}\left(d_{\mathbf{S}}\right)\right)=\Omega\left(\frac{\left(d_{\mathbf{S}}\right)^{2}}{\log n \cdot n^{\frac{\delta}{2}}}\right)$.

(2) When $d_{\mathbf{S}}=O\left(n^{\frac{\delta}{2}}\right)$

Recall that the velocity of nodes under the DRDMM, say $v(n)$, is of order $\Theta(1)$. When $d_{\mathbf{S}}=O\left(n^{\frac{\delta}{2}}\right)$, it holds that $\mathbf{E}\left(\tau_{E}^{\mathrm{d}}\left(d_{\mathbf{S}}\right)\right)=\Omega\left(\frac{d_{\mathbf{S}}}{v(n)}\right)$, which directly proves that $\mathbf{E}\left(\tau_{E}^{\mathrm{d}}\left(d_{\mathbf{S}}\right)\right)=\Omega\left(d_{\mathbf{S}}\right)$.

(3) When $d_{\mathbf{S}}=\Omega\left(n^{\frac{\delta}{2}}\right)$ and $d_{\mathbf{S}}=O\left(n^{\frac{\delta}{2}} \cdot \sqrt{\log n}\right)$

It is clear that $\tau_{E}^{\mathrm{d}}\left(d_{\mathbf{S}}\right)=\Omega\left(L_{\mathrm{p}}^{\mathrm{d}}\right)$ when $d_{\mathbf{S}}=\Omega\left(n^{\frac{\delta}{2}}\right)$. By $L_{\mathrm{p}}^{\mathrm{d}}=\Theta\left(n^{\frac{\delta}{2}}\right)$, we get that $\mathbf{E}\left(\tau_{E}^{\mathrm{d}}\left(d_{\mathbf{S}}\right)\right)=\Omega\left(n^{\frac{\delta}{2}}\right)$.

\subsubsection{Duration of Waiting Intervals}

Let $\tau_{W}^{\mathrm{h}}\left(d_{\mathbf{S}}\right)$ and $\tau_{W}^{\mathrm{d}}\left(d_{\mathbf{S}}\right)$ denote the durations of waiting intervals under a given communication strategy $\mathbf{S}$ for the HRWMM and DRDMM, respectively. Then, we have

Lemma 6: Under the HRWMM and the strategy $\mathbf{S}$ with a critical distance $d_{\mathbf{S}}$, with high probability, $\mathbf{E}\left(\tau_{W}^{\mathrm{h}}\left(d_{\mathbf{S}}\right)\right)$ is of order

$$
\left\{\begin{array}{lll}
\Omega\left(\frac{n^{1-\gamma}}{\log n}\right) & \text { when } & d_{\mathbf{S}}:[1, \sqrt{n}), \\
\Theta\left(\frac{n}{\left(d_{\mathbf{S})^{2}}\right.}+\frac{\log n}{n^{\gamma-1}}\right) & \text { when } & d_{\mathbf{S}}:[1, \sqrt{n}) \cap\left[1, n^{\frac{\gamma}{2}}\right], \\
\Theta(1) & \text { when } & d_{\mathbf{S}}=\Theta(\sqrt{n}) .
\end{array}\right.
$$

Proof: For the HRWMM, let $\tau_{H}^{\mathrm{h}}$ denote the time required by nodes to enter the same cell from their initial random and uniformly distributed positions, i.e., the first hitting time (Definition B.1 in Appendix B); and let $\tau_{R}^{\mathrm{h}}$ denote the time between successive hitting states, i.e., the return time (Definition B.1 in Appendix B). Now, we analyze the order of $\tau_{W}^{\mathrm{h}}\left(d_{\mathbf{S}}\right)$, i.e., the duration of waiting intervals in a two-hop strategy $\mathbf{S}$ under the HRWMM.

In the following analysis, $\varphi_{i}^{\mathrm{h}}, i \geq 1$, denote some proper constants whose specific values have no impact on the order of our results.

Note that the HRWMM is based on the lattice (torus) $\mathbb{L}\left(n, n^{\gamma}\right)$. According to Corollary B.1 in Appendix B, it holds with high probability that

$$
\mathbf{E}\left(\tau_{H}^{\mathrm{h}}\right)=\Theta\left(n^{1-\gamma} \cdot \log n\right) ; \quad \mathbf{E}\left(\tau_{R}^{\mathrm{h}}\right)=\Theta\left(n^{1-\gamma}\right) .
$$

(1) When $d_{\mathbf{S}}:[1, \sqrt{n}) \cap\left[1, n^{\frac{\gamma}{2}}\right]$

$\tau_{W}^{\mathrm{h}}\left(d_{\mathbf{S}}\right)=\sum_{k=0}^{\infty}\left(\tau_{H}^{\mathrm{h}}+k \cdot \tau_{R}^{\mathrm{h}}\right) \cdot\left(1-\varphi_{2}^{\mathrm{h}} \frac{\left(d_{\mathbf{S}}\right)^{2}}{n^{\gamma}}\right)^{k} \cdot \varphi_{1}^{\mathrm{h}} \cdot \frac{\left(d_{\mathbf{S}}\right)^{2}}{n^{\gamma}}$, where $0^{0}:=1$. Then,

$$
\begin{aligned}
& \mathbf{E}\left(\tau_{W}^{\mathrm{h}}\left(d_{\mathbf{S}}\right)\right) \\
& =\frac{\varphi_{1}^{\mathrm{h}}\left(d_{\mathbf{S}}\right)^{2}}{n^{\gamma}} \cdot\left(\mathbf{E}\left(\tau_{H}^{\mathrm{h}}\right) \cdot \frac{n^{\gamma}}{\left(d_{\mathbf{S}}\right)^{2}}+\mathbf{E}\left(\tau_{R}^{\mathrm{h}}\right) \cdot \frac{n^{4 \frac{\gamma}{2}}-\left(d_{\mathbf{S}}\right)^{2} \cdot n^{\gamma}+\left(d_{\mathbf{S}}\right)^{4}}{\left(d_{\mathbf{S}}\right)^{4}}\right) \\
& =\Theta\left(\mathbf{E}\left(\tau_{H}^{\mathrm{h}}\right)\right)+\Theta\left(\mathbf{E}\left(\tau_{R}^{\mathrm{h}}\right) \cdot \frac{n^{\gamma}}{\left(d_{\mathbf{S}}\right)^{2}}\right) .
\end{aligned}
$$

Hence, we get that

$$
\mathbf{E}\left(\tau_{W}^{\mathrm{h}}\left(d_{\mathbf{S}}\right)\right)=\Theta\left(\frac{n}{\left(d_{\mathbf{S}}\right)^{2}}+n^{1-\gamma} \cdot \log n\right) .
$$

(2) When $d_{\mathbf{s}}=\Theta(\sqrt{n})$

$$
\mathbf{E}\left(\tau_{W}^{\mathrm{h}}\left(d_{\mathbf{S}}\right)\right)=\Theta(1) .
$$

(3) A general lower bound for the regime $d_{\mathbf{S}}:[1, \sqrt{n})$

Since the deployment region is assumed to be a twodimensional torus, it follows that

$$
\mathbf{E}\left(\tau_{W}^{\mathrm{h}}\left(d_{\mathbf{S}}\right)\right)=\Theta\left(\mathbf{E}\left(\tau_{C}^{\mathrm{h}}\left(\varphi_{3}^{\mathrm{h}} \sqrt{n}-d_{\mathbf{S}}\right)\right)\right) .
$$

Then, according to Lemma 4 , we obtain that for $d_{\mathbf{S}}:[1, \sqrt{n})$

$$
\mathbf{E}\left(\tau_{W}^{\mathrm{h}}\left(d_{\mathbf{S}}\right)\right)=\Omega\left(\frac{n^{1-\gamma}}{\log n}\right),
$$

which completes the proof.

Lemma 7: Under the DRDMM and the strategy $\mathbf{S}$ with a critical distance $d_{\mathbf{S}}$, with high probability, $\mathbf{E}\left(\tau_{W}^{\mathrm{d}}\left(d_{\mathbf{S}}\right)\right)$ is of order

$$
\left\{\begin{array}{lll}
\Omega\left(\frac{n^{1-\frac{\delta}{2}}}{\log n}\right) & \text { when } & d_{\mathbf{S}}:[1, \sqrt{n}), \\
\Theta\left(n^{1-\frac{\delta}{2}} \log n+\frac{n}{d_{\mathbf{S}}}\right) & \text { when } & d_{\mathbf{S}}:[1, \sqrt{n}) \cap\left[1, n^{\frac{\delta}{2}}\right], \\
\Theta(1) & \text { when } & d_{\mathbf{S}}=\Theta(\sqrt{n}) .
\end{array}\right.
$$

Proof: For the DRDMM, let $\tau_{H}^{\mathrm{d}}$ denote the time required by nodes to enter the same cell from their initial random and uniformly distributed positions, i.e., the first hitting time (Definition B.1 in Appendix B); and let $\tau_{R}^{\mathrm{d}}$ denote the time between successive hitting states, i.e., the return time (Definition B.1 in Appendix B). Now, we analyze the order of $\tau_{W}^{\mathrm{d}}\left(d_{\mathbf{S}}\right)$, i.e., 
the duration of waiting intervals in a given two-hop strategy $\mathbf{S}$ under the DRDMM according to the value of $\mathbb{L}\left(n,\left(d_{\mathbf{S}}\right)^{2}\right)$.

In the following analysis, $\varphi_{i}^{\mathrm{d}}, i \geq 1$, denote some proper constants whose specific values do not change our results in the sense of order.

Recall that the DRDMM is based on a lattice (torus) $\mathbb{L}\left(n, n^{\delta}\right)$, and the duration of each motion phase is $\varphi_{1}^{\mathrm{d}} \cdot n^{\frac{\delta}{2}}$ (Fig. 4). According to Corollary B.1 in Appendix B, with high probability, it holds that

$$
\mathbf{E}\left(\tau_{H}^{\mathrm{d}}\right)=\Theta\left(n^{1-\delta} \cdot \log n\right) ; \mathbf{E}\left(\tau_{R}^{\mathrm{d}}\right)=\Theta\left(n^{1-\delta}\right)
$$

(1) When $d_{\mathbf{S}}:[1, \sqrt{n}) \cap\left[1, n^{\frac{\delta}{2}}\right]$

Similar to Case 1 in the proof of Lemma 6, it follows that

$\mathbf{E}\left(\tau_{W}^{\mathrm{d}}\left(d_{\mathbf{S}}\right)\right)=\varphi_{1}^{\mathrm{d}} \cdot n^{\frac{\delta}{2}} \cdot\left(\Theta\left(\mathbf{E}\left(\tau_{H}^{\mathrm{d}}\right)\right)+\Theta\left(\frac{n^{\frac{\delta}{2}}}{d_{\mathbf{S}}}\right) \cdot \Theta\left(\mathbf{E}\left(\tau_{R}^{\mathrm{d}}\right)\right)\right)$

where $\Theta\left(d_{\mathbf{S}} / n^{\frac{\delta}{2}}\right)$ is the probability that two nodes located in the same cell can meet within a distance of $d_{\mathbf{S}}$ during a motion phase. We remark that it is different from the case in the HRWMM, due to the continuous motion of nodes. Then,

$$
\mathbf{E}\left(\tau_{W}^{\mathrm{d}}\left(d_{\mathbf{S}}\right)\right)=\Theta\left(n^{1-\frac{\delta}{2}} \log n+\frac{n}{d_{\mathbf{S}}}\right)
$$

(2) When $d_{\mathbf{S}}=\Theta(\sqrt{n})$

$$
\mathbf{E}\left(\tau_{W}^{\mathrm{d}}\left(d_{\mathbf{S}}\right)\right)=\Theta(1)
$$

(3) A general lower bound in the regime $d_{\mathbf{S}}:[1, \sqrt{n})$

Similar to Case 3 in the proof of Lemma 6, it follows that

$$
\mathbf{E}\left(\tau_{W}^{\mathrm{d}}\left(d_{\mathbf{S}}\right)\right)=\Theta\left(\mathbf{E}\left(\tau_{C}^{\mathrm{d}}\left(\varphi_{1}^{\mathrm{d}} \sqrt{n}-d_{\mathbf{S}}\right)\right)\right)
$$

Then, according to Lemma 5, we get that

$$
\mathbf{E}\left(\tau_{W}^{\mathrm{d}}\left(d_{\mathbf{S}}\right)\right)=\Omega\left(\frac{n^{1-\frac{\delta}{2}}}{\log n}\right) \text { for } d_{\mathbf{S}}:[1, \sqrt{n}) .
$$

Combining the three cases above, we get this lemma.

\section{Capacity and Delay Analysis}

Depending on the critical distance $d_{\mathbf{S}}:[1, \sqrt{n}]$ of a given two-hop strategy $\mathbf{S}$ under the generalized physical model with a power attenuation exponent $\alpha>2$, we derive the asymptotic per-session capacity $\lambda(\mathbf{S}, n)$ and the average delay $\mathbf{E}(D(\mathbf{S}, n))$ under both the HRWMM and DRDMM. As commonly done in the literature, we set the number of sessions to $K=n$, although all definitions, strategies and analysis proposed above are applicable for general values of $K$.

\subsection{Capacity and Delay under the HRWMM}

Theorem 1: Under the generalized physical model and HRWMM, by using the two-hop strategy $\mathbf{S}$ with a critical distance $d_{\mathbf{S}}$, the achievable average throughput, denoted by $\lambda^{\mathrm{h}}(\mathbf{S}, n)$, is of order

$$
\left\{\begin{array}{lll}
\Omega\left(\frac{\log n \cdot n^{\gamma}}{\left(d_{\mathbf{S}}\right)^{2}}\right) & \text { when } & d_{\mathbf{S}}:\left[n^{\frac{\gamma}{2}} \sqrt{\log n}, \sqrt{n}\right], \\
\Theta(1) & \text { when } & d_{\mathbf{S}}:\left[1, n^{\frac{\gamma}{2}} \sqrt{\log n}\right] \cap[1, \sqrt{n}] .
\end{array}\right.
$$

The average delay, denoted by $\mathbf{E}\left(D^{\mathrm{h}}(\mathbf{S}, n)\right)$, is of the order

$$
\left\{\begin{array}{lll}
\Theta\left(\frac{n}{\left(d_{\mathbf{S}}\right)^{2}}+\frac{\log n}{n^{\gamma-1}}\right) & \text { when } & d_{\mathbf{S}}:\left[1, n^{\frac{\gamma}{2}}\right] \cap[1, \sqrt{n}), \\
\Omega\left(\frac{n^{1-\gamma}}{\log n}\right) & \text { when } & d_{\mathbf{S}}:\left[n^{\frac{\gamma}{2}}, \sqrt{n}\right) \\
O\left(\frac{n^{1-\gamma}}{\log n}+1\right) & \text { when } & d_{\mathbf{S}}=\Theta(\sqrt{n}) .
\end{array}\right.
$$

Proof: Please refer to Appendix C.1.

Specifically, we have:

(a) When $\gamma=0$, as illustrated in Fig. 6(a),
$\lambda(\mathbf{S}, n)=\left\{\begin{array}{lll}\Theta(1) & \text { when } & d_{\mathbf{S}}:[1, \sqrt{\log n}], \\ \Omega\left(\frac{\log n}{\left(d_{\mathbf{S}}\right)^{2}}\right) & \text { when } & d_{\mathbf{S}}:(\sqrt{\log n}, \sqrt{n}] .\end{array}\right.$
$\mathbf{E}(D(\mathbf{S}, n))=\left\{\begin{array}{lll}\Theta(n \log n) & \text { when } & d_{\mathbf{S}}=\Theta(1), \\ \Omega\left(\frac{n}{\log n}\right) & \text { when } & d_{\mathbf{S}}:(1, \sqrt{n}), \\ O\left(\frac{n}{\log n}\right) & \text { when } & d_{\mathbf{S}}=\Theta(\sqrt{n}) .\end{array}\right.$

(b) When $0<\gamma<1$, as illustrated in Fig. 6(b),

$$
\begin{aligned}
& \lambda(\mathbf{S}, n)=\left\{\begin{array}{lll}
\Theta(1) & \text { when } \quad d_{\mathbf{S}}:\left[1, \sqrt{n^{\gamma} \log n}\right], \\
\Omega\left(\frac{\log n \cdot n^{\gamma}}{\left(d_{\mathbf{S}}\right)^{2}}\right) & \text { when } & d_{\mathbf{S}}:\left(\sqrt{n^{\gamma} \log n}, \sqrt{n}\right] .
\end{array}\right. \\
& \mathbf{E}(D(\mathbf{S}, n))=\left\{\begin{array}{lll}
\Theta\left(\frac{n}{\left(d_{\mathbf{S}}\right)^{2}}\right) & \text { when } & d_{\mathbf{S}}:\left[1, \sqrt{\frac{n^{\gamma}}{\log n}}\right], \\
\Theta\left(\frac{\log n}{n^{\gamma-1}}\right) & \text { when } & d_{\mathbf{S}}:\left[\sqrt{\frac{n^{\gamma}}{\log n}}, n^{\frac{\gamma}{2}}\right], \\
\Omega\left(\frac{n^{1-\gamma}}{\log n}\right) & \text { when } & d_{\mathbf{S}}:\left(n^{\frac{\gamma}{2}}, \sqrt{n}\right), \\
O\left(\frac{n^{1-\gamma}}{\log n}\right) & \text { when } & d_{\mathbf{S}}=\Theta(\sqrt{n}) .
\end{array}\right.
\end{aligned}
$$
$6(c)$,

(c) When $\gamma=1, \lambda(\mathbf{S}, n)=\Theta(1)$, as illustrated in Fig.

$\mathbf{E}(D(\mathbf{S}, n))=\left\{\begin{array}{lll}\Theta\left(\frac{n}{\left(d_{\mathbf{S}}\right)^{2}}\right) & \text { when } & d_{\mathbf{S}}:\left[1, \sqrt{\frac{n}{\log n}}\right), \\ \Theta(\log n) & \text { when } & d_{\mathbf{S}}:\left[\sqrt{\frac{n}{\log n}}, \sqrt{n}\right), \\ \Theta(1) & \text { when } & d_{\mathbf{S}}=\Theta(\sqrt{n}) .\end{array}\right.$

Now, we examine the implications of the results for the HRWMM.

- Under the classical two-hop strategy, to achieve the capacity of order $\Theta(1)$ for dense networks, the critical distance is $d_{\mathbf{S}}=\Theta\left(\frac{1}{\sqrt{n}}\right)$ [8]. Then, it is intuitive that by a simple scaling extension from dense networks to extended networks, i.e., by letting $d_{\mathbf{S}}=\Theta(1)$, the capacity of order $\Theta(1)$ is achievable. Here we prove that the tight upper bound of $d_{\mathbf{S}}$ for the optimal capacity under the generalized physical model is $\Theta\left(\min \left\{n^{\frac{\gamma}{2}} \sqrt{\log n}, \sqrt{n}\right\}\right)$ (The feasible region is $\left.d_{\mathbf{S}}:\left[1, \min \left\{n^{\frac{\gamma}{2}} \sqrt{\log n}, \sqrt{n}\right\}\right]\right)$. 

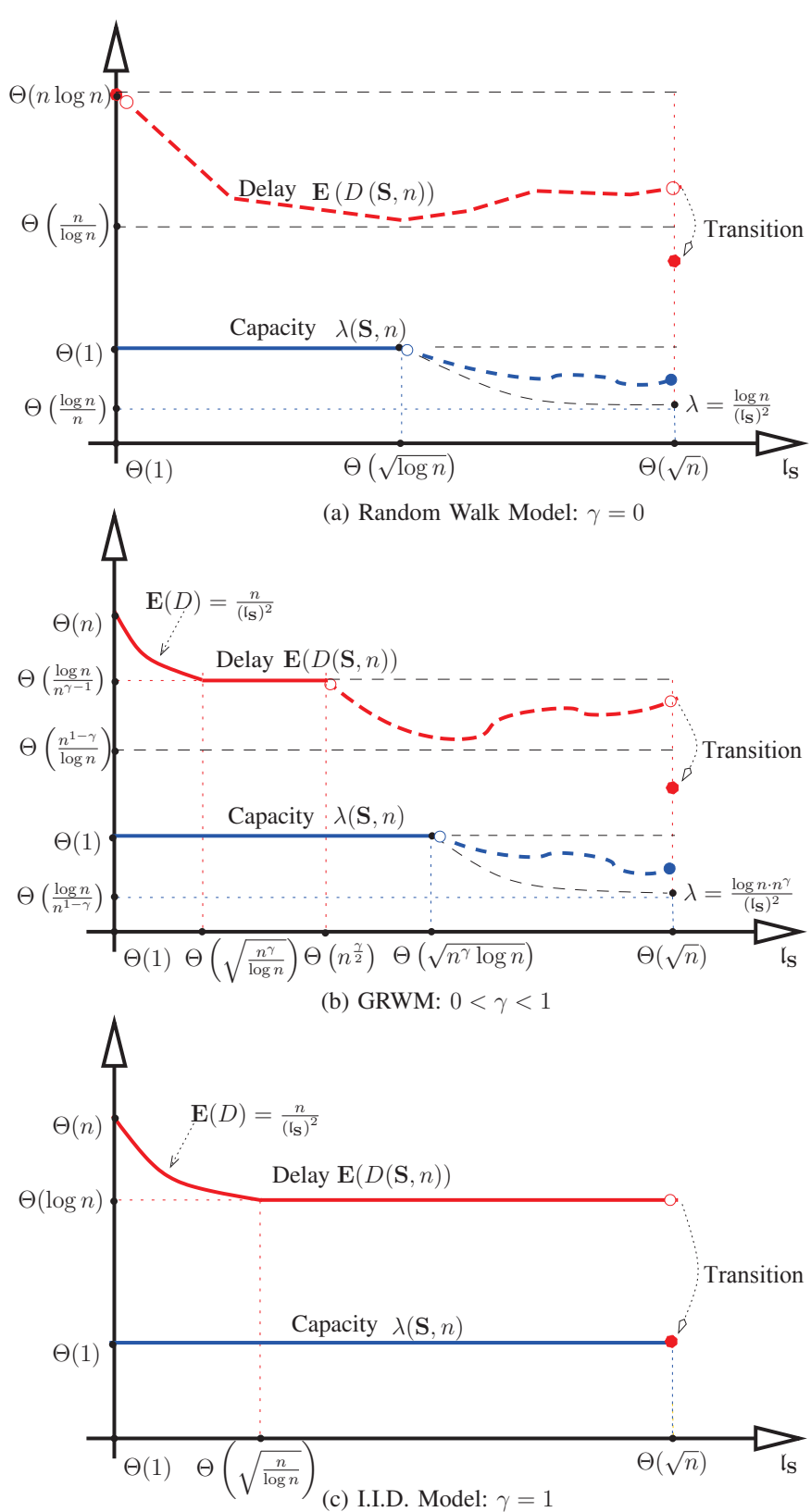

Fig. 6. Achievable capacity and delay depending on the degree of freedom $\gamma \in[0,1]$. The solid curves represent the functions of the achievable capacities and delays with respect to the parameter $d_{\mathbf{s}}$. The dashed curves represent the functions of the achievable capacities and delays whose lower and upper bounds can be derived but the exact forms cannot be determined.

- For any $\gamma \in[0,1]$, when the optimal capacity of order $\Theta(1)$ is sustained, the corresponding delay is non-increasing with $d_{\mathbf{S}}$; when $d_{\mathbf{S}}=\Theta(\sqrt{n})$, a transition of delay occurs. This gain is obtained by the advantage of rate adaptation under the generalized physical model. For $\gamma=1$, i.e., the i.i.d. model, there is a transition of the optimal delay: the capacity and delay can be simultaneously achieved to the optimal order, i.e., $\Theta(1)$, by the simple two-hop strategy. Please refer to Fig. 6(c) and Fig. 7 for the illustrations. This ideal result comes

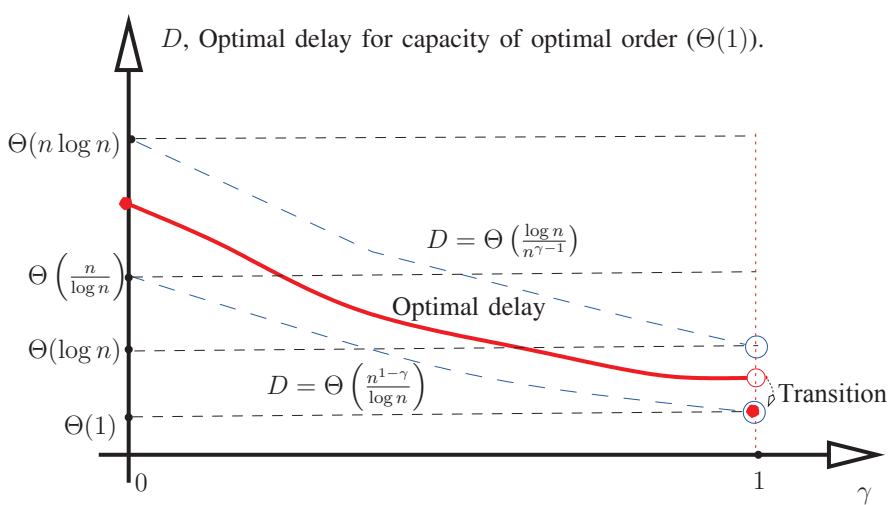

Fig. 7. Optimal delay depending on the degree of freedom $\gamma$. The solid curve (including the "singletons", i.e., isolated nodes) denotes the function of optimal delay in terms of $\gamma$; the dashed curves denote the upper and lower bounds of this function.

from the extreme high freedom $(\gamma=1)$ of nodes under the i.i.d. mobility model and the advantages of the general physical model. As a matter of fact, this result breaks the limitations of capacity-delay tradeoffs derived under the protocol model and the physical model in the literature, [24]-[31]. We state that the transition is the result of the particularity of the i.i.d. model. Recall that for any node, its current position is independent of its positions in the adjacent time slots under the i.i.d. mobility model. This means that in the extended network the velocity of each node under i.i.d. model is increasing to infinity (of order $\Theta(\sqrt{n})$, as the network size $n \rightarrow \infty$. We emphasize that this particularity of the i.i.d. mobility model contributes to this surprising result. To some extent, it indicates that the i.i.d. model which is a very special one, is not a proper model in analyzing the performance of realistic mobile networks.

- Furthermore, for the delay, in the first regime of Equation (10), i.e., $d_{\mathbf{S}}:\left[1, n^{\frac{\gamma}{2}}\right] \cap[1, \sqrt{n})$, by using the tight bound, we get that the delay is inversely proportional to $\left(d_{\mathbf{S}}\right)^{2}$ when $d_{\mathbf{S}}:\left[1, \frac{n^{\frac{\gamma}{2}}}{\sqrt{\log n}}\right]$, and becomes invariable when $d_{\mathbf{S}}$ is beyond the threshold of order $\Theta\left(\frac{n^{\frac{\gamma}{2}}}{\sqrt{\log n}}\right)$. For the other two regimes, it is an interesting future work to derive tight bounds if they exist, which can possibly enhance the insights of the issue.

Under the premise of ensuring the optimal capacity of order $\Theta(1)$, when $0<\gamma \leq 1$, the optimal delay under the GphyM is smaller than that under the protocol model or the physical model; and when $\gamma=0$ (random walk model), it is no larger than the delay under the protocol model or the physical model. We give the corresponding illustrations in Figs. 1(a)$1(\mathrm{c})$.

\subsection{Capacity and Delay under the DRDMM}

Theorem 2: Under the generalized physical model and DRDMM, by applying the two-hop strategy $\mathbf{S}$ with a critical distance $d_{\mathbf{S}}$, the achievable average throughput, denoted by $\lambda^{\mathrm{d}}(\mathbf{S}, n)$, is of order 


$$
\begin{cases}\Omega\left(\frac{\log n \cdot n^{\frac{\delta}{2}}}{\left(d_{\mathbf{S}}\right)^{2}}\right) & \text { when } d_{\mathbf{S}}:\left[n^{\frac{\delta}{2}} \sqrt{\log n}, \sqrt{n}\right], \\ \Omega\left(\frac{1}{n^{\frac{\delta}{2}}}\right) & \text { when } d_{\mathbf{S}}:\left[n^{\frac{\delta}{2}}, n^{\frac{\delta}{2}} \sqrt{\log n}\right] \cap[1, \sqrt{n}, \\ \Omega\left(\frac{1}{d_{\mathbf{S}}}\right) & \text { when } d_{\mathbf{S}}:\left[1, n^{\frac{\delta}{2}}\right] .\end{cases}
$$

The average delay, denoted by $\mathbf{E}\left(D^{\mathrm{d}}(\mathbf{S}, n)\right)$, is of order

$$
\begin{cases}\Theta\left(\frac{n}{\left(d_{\mathbf{S}}\right)^{2}}+\frac{\log n}{n^{\frac{\delta}{2}-1}}\right) & \text { when } d_{\mathbf{S}}:\left[1, n^{\frac{\delta}{2}}\right] \cap[1, \sqrt{n}), \\ \Omega\left(\frac{n^{1-\frac{\delta}{2}}}{\log n}\right) & \text { when } d_{\mathbf{S}}:\left[n^{\frac{\delta}{2}}, \sqrt{n}\right), \\ O\left(\frac{n^{1-\frac{\delta}{2}}}{\log n}\right) & \text { when } d_{\mathbf{S}}=\Theta(\sqrt{n}) .\end{cases}
$$

Proof: Please refer to Appendix C.2.

Specifically, we have:

(a) When $\delta=0$, as illustrated in Fig. 8(a),

$$
\begin{aligned}
& \lambda(\mathbf{S}, n)= \begin{cases}\Omega(1) & \text { when } d_{\mathbf{S}}:[1, \sqrt{\log n}], \\
\Omega\left(\frac{\log n}{\left(d_{\mathbf{S}}\right)^{2}}\right) & \text { when } d_{\mathbf{S}}:[\sqrt{\log n}, \sqrt{n}] .\end{cases} \\
& \mathbf{E}(D(\mathbf{S}, n))= \begin{cases}\Theta(n \log n) & \text { when } d_{\mathbf{S}}:[1, \sqrt{n}), \\
O\left(\frac{n}{\log n}\right) & \text { when } d_{\mathbf{S}}=\Theta(\sqrt{n}) .\end{cases}
\end{aligned}
$$

(b) When $0<\delta<1$, as illustrated in Fig. 8(b),

$$
\begin{aligned}
& \lambda(\mathbf{S}, n)= \begin{cases}\Omega\left(\frac{1}{d_{\mathbf{S}}}\right) & \text { when } d_{\mathbf{S}}:\left[1, n^{\frac{\delta}{2}}\right], \\
\Omega\left(\frac{1}{n^{\frac{\delta}{2}}}\right) & \text { when } d_{\mathbf{S}}:\left[n^{\frac{\delta}{2}}, n^{\frac{\delta}{2}} \sqrt{\log n}\right], \\
\Omega\left(\frac{\log n \cdot n^{\frac{\delta}{2}}}{\left(d_{\mathbf{S}}\right)^{2}}\right) & \text { when } d_{\mathbf{S}}:\left[n^{\frac{\delta}{2}} \sqrt{\log n}, \sqrt{n}\right] .\end{cases} \\
& \mathbf{E}(D(\mathbf{S}, n))= \begin{cases}\Theta\left(\frac{n}{\left(d_{\mathbf{S}}\right)^{2}}\right) & \text { when } d_{\mathbf{S}}:\left[1, \frac{n^{\frac{\delta}{4}}}{\sqrt{\log n}}\right], \\
\Theta\left(\frac{\log n}{n^{\frac{\delta}{2}-1}}\right) & \text { when } d_{\mathbf{S}}:\left[\frac{n^{\frac{\delta}{4}}}{\sqrt{\log n}}, n^{\frac{\delta}{2}}\right], \\
\Omega\left(\frac{n^{1-\frac{\delta}{2}}}{\log n}\right) & \text { when } d_{\mathbf{S}}:\left[n^{\frac{\delta}{2}}, \sqrt{n}\right), \\
O\left(\frac{n^{1-\frac{\delta}{2}}}{\log n}\right) & \text { when } d_{\mathbf{S}}=\Theta(\sqrt{n}) .\end{cases}
\end{aligned}
$$

(c) When $\delta=1$, as illustrated in Fig. $8(\mathrm{c}), \lambda(\mathbf{S}, n)=$ $\Omega\left(\frac{1}{d_{\mathbf{S}}}\right)$ for $d_{\mathbf{S}}:[1, \sqrt{n}]$,

$\mathbf{E}(D(\mathbf{S}, n))= \begin{cases}\Theta\left(\frac{n}{\left(d_{\mathbf{S}}\right)^{2}}\right) & \text { when } d_{\mathbf{S}}:\left[1, \frac{\sqrt[4]{n}}{\sqrt{\log n}}\right], \\ \Theta(\sqrt{n} \cdot \log n) & \text { when } d_{\mathbf{S}}:\left(\frac{\sqrt[4]{n}}{\sqrt{\log n}}, \sqrt{n}\right), \\ O\left(\frac{\sqrt{n}}{\log n}\right) & \text { when } d_{\mathbf{S}}=\Theta(\sqrt{n}) .\end{cases}$

Now, we examine the implications of the results for the DRDMM.

- For the cases of $0<\delta \leq 1$, including the random waypoint model, one has to let $d_{\mathbf{S}}=\Theta(1)$ in order to achieve the optimal capacity of order $\Theta(1)$. For the discrete Brownian mobility model, i.e., the case of $\delta=0$, the optimal capacity is achievable when $d_{\mathbf{S}}:[1, \sqrt{\log n}]$, as shown in Table. 3 .

- The bound on the delay is tight in the regime of $d_{\mathbf{S}}$ : $\left[1, n^{\frac{\delta}{2}}\right] \cap[1, \sqrt{n})$. The delay is inversely proportional to $\left(d_{\mathbf{S}}\right)^{2}$
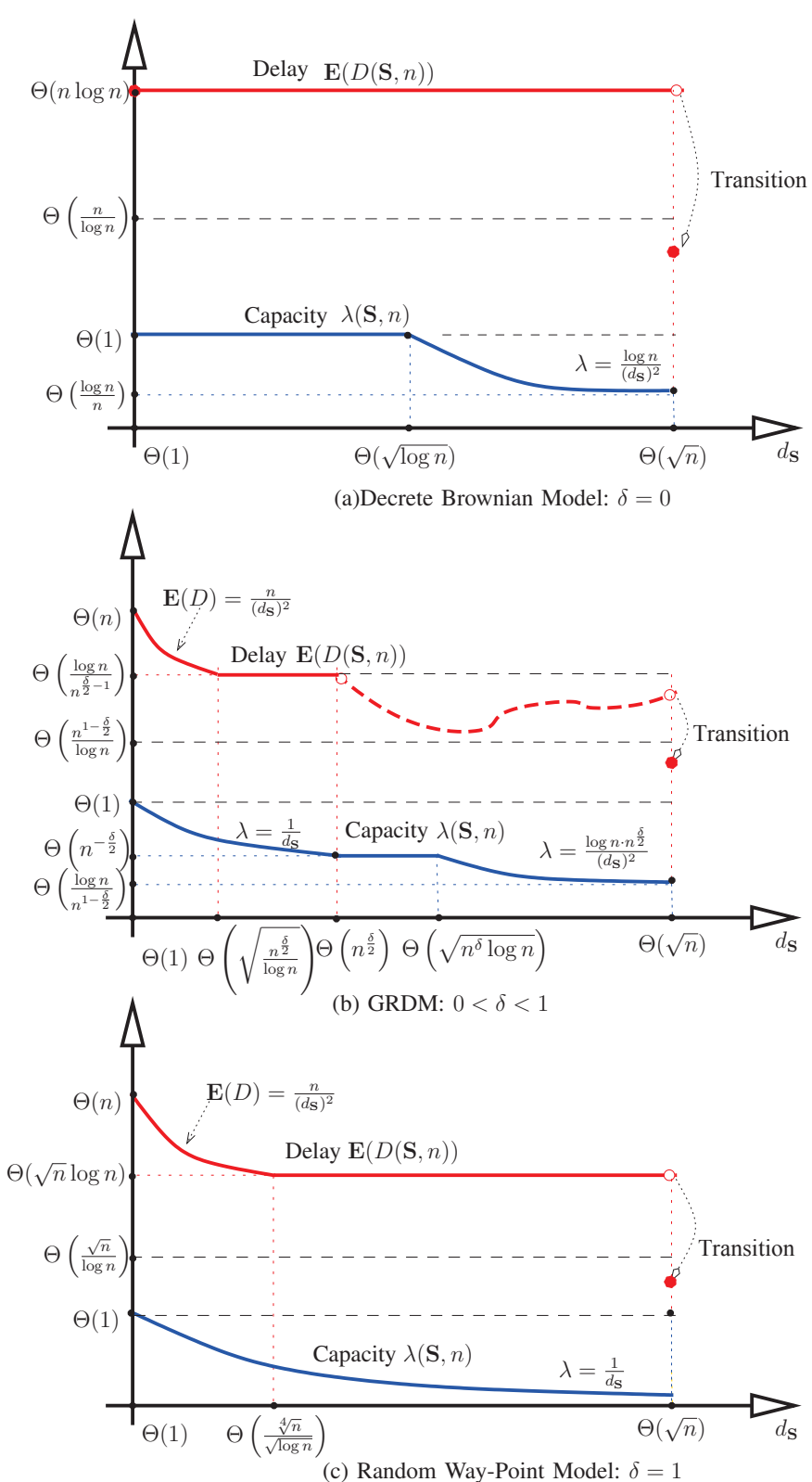

(c) Random Way-Point Model: $\delta=1$

Fig. 8. Achievable capacity and delay depending on the degree of freedom $\delta \in[0,1]$. The solid curves represent the functions of the achievable capacities and delays with respect to the parameter $d_{\mathbf{S}}$. The dashed curve represents the function of the achievable delay whose lower and upper bounds can be derived but exact form cannot be determined.

when $d_{\mathbf{S}}:\left[1, \frac{n^{\frac{\delta}{4}}}{\sqrt{\log n}}\right]$, and becomes invariable when $d_{\mathbf{S}}$ is beyond the threshold $\Theta\left(\frac{n^{\frac{\delta}{4}}}{\sqrt{\log n}}\right)$.

- Under the premise of ensuring the optimal capacity of order $\Theta(1)$, when $0<\delta \leq 1$, the optimal delay under the GphyM is the same as that under the protocol model or the physical model; and when $\delta=0$ (discrete Brownian model), it is no larger than the delay under the protocol model or the physical model. We provide the corresponding illustrations in 
TABLE 3

Range of $d_{\mathbf{S}}$ for the optimal-order capacity.

\begin{tabular}{c|c}
\hline & Range of $d_{\mathbf{S}}$ \\
\hline \hline HRWMM & $d_{\mathbf{S}}:\left[1, \min \left\{n^{\frac{\gamma}{2}} \sqrt{\log n}, \sqrt{n}\right\}\right]$ \\
\hline DRDMM & $d_{\mathbf{S}}:[1, \sqrt{\log n}]$ if $\delta=0 ; \quad d_{\mathbf{S}}=\Theta(1)$ if $0<\delta \leq 1$ \\
\hline
\end{tabular}

Fig. 1(d)-(f).

\section{IntUitive Explanations of The Results}

In this section, we provide a nonrigorous but intuitive analysis for the impacts of rate adaptation and mobility models on the capacity-delay tradeoffs for MANETs.

\subsection{Key Points for Improving Tradeoffs}

In MANETs, there are generally two ways to send a packet from the source to its destination(s), i.e., wireless transmission and physical transportation on a carrier. Accordingly, the sum of travel distances of packets, denoted by $L$, can be divided into two parts: the sum of distances covered by wireless transmissions and the sum of distances covered by carrier movement, denoted by $L_{T}$ and $L_{M}$, respectively. It holds that $L=L_{T}+L_{M}$. Intuitively, for improving capacity, the distances by wireless transmissions should be reduced, while for decreasing delay, the distances by carrier movements should be reduced. However, as a general rule, both types of distances cannot be simultaneously decreased, which potentially results in a tradeoff between the network capacity and delay. For instance, in the pioneering work [8], under the i.i.d. mobility model, the optimal capacity of order $\Theta(1)$ is achieved by minimizing the distances covered by wireless transmissions, consequently maximizing the distances covered by carrier movements, at the cost of a huge delay of order $\Theta(n)$.

In most strategies for improving the capacity-delay tradeoffs in MANETs, e.g., the packet redundancy scheme [11], [18], there is a common argument, that is, "less loss" of capacity in exchange for "more gain" of delay. Under this argument, we aim to investigate whether the capacity-delay tradeoffs in MANETs can be improved by permitting adaptive rate wireless transmissions.

\subsection{Intuitive Justification of the Gains of Rate Adap- tation}

When longer-distance wireless transmissions are permitted by introducing adaptive-rate communication model, the distances covered by the physical movement of the carriers can be reduced with high probability, which results in the decrease of waiting delay, with possible loss of capacity. Based on this thought, we demonstrate whether relay schemes with longer wireless links of an appropriate distance can improve capacitydelay tradeoffs for MANETs. In other words, it indicates whether we can get "more gain" of delay at the expense of "less loss" of capacity. It shows that the answer depends on the property of mobility models. For the mobility models under which the movements of nodes are relatively "rapid", e.g., the i.i.d. model, compared with strategies under the fixed-rate communication model, there exist larger thresholds of critical distances below which the capacity is not reduced heavily, and even the optimal capacity can be still sustained. The reason for such results lies in the fact that the data transmitted via long-distance links are indeed infinitesimal relative to those via short links when the link rate changes with the link length under the adaptive-rate communication model, which can explain why improvements on capacity-delay tradeoffs for some mobility models can be achieved by the rate adaptation.

\section{Conclusion}

In this paper, we initiated an investigation of the impact of adaptive-rate communication model on capacity-delay tradeoffs in MANETs under some classical mobility models. We derived the optimal delay for the optimal unicast capacity by using the well-known two-hop relay policy, and made it clear how the capacity-delay tradeoffs in MANETs vary under the different mobility models when the rate adaptation is introduced.

There are some limitations of our work that are left for the future research:

(1) There remain gaps between the lower and upper bounds on capacity and delay for some regimes. It is necessary to derive tight bounds in the whole regime and provide more complete and conclusive results.

(2) In order to concentrate on stressing new insights of the impact of rate adaptation, we constrained the strategies to the type of simple threshold-based two-hop relaying schemes in this work. An important work is to extend our results by adopting some advanced relay techniques, such as replication and network-layer cooperation policies.

(3) We only considered unicast sessions in this work. It should be interesting to extend our results to other traffic sessions, e.g., multicast, broadcast, convergecast, anycast and manycast.

\section{ACKNOWLEDGEMENT}

The research of authors is partially supported by the the National Natural Science Foundation of China (NSFC) under grant No. 61202383, No. 61170216, No. 61228202, the National Basic Research Program of China (973 Program) under grant No. 2010CB328101, NSF CNS-0832120, NSF CNS1035894, NSF ECCS-1247944, NSF ECCS-1343306. Any opinions, findings, conclusions, or recommendations expressed in this paper are those of author(s) and do not necessarily reflect the views of the funding agencies (NSF and NSFC).

\section{References}

[1] P. Gupta and P. R. Kumar, "The capacity of wireless networks," IEEE Trans. on Information Theory, vol. 46, no. 2, pp. 388-404, 2000. 
[2] O. Leveque and I. Telatar, "Information-theoretic upper bounds on the capacity of large extended ad hoc wireless networks," IEEE Trans. on Information Theory, vol. 51, no. 3, pp. 858-865, 2005.

[3] A. Özgür, R. Johari, D. N. C. Tse, and O. Lévêque, "Informationtheoretic operating regimes of large wireless networks," IEEE Trans. on Information Theory, vol. 56, no. 1, pp. 427-437, 2010.

[4] A. Özgür, O. Lévêque, and D. N. C. Tse, "Hierarchical cooperation achieves optimal capacity scaling in ad hoc networks," IEEE Trans. on Information Theory, vol. 53, no. 10, pp. 3549-3572, 2007.

[5] R. Groenevelt, P. Nain, and G. Koole, "The message delay in mobile ad hoc networks," Performance Evaluation, vol. 62, no. 1, pp. 210-228, 2005.

[6] J. Zhao and G. Cao, "Vadd: Vehicle-assisted data delivery in vehicular ad hoc networks," IEEE Trans. on Vehicular Technology, vol. 57, no. 3, pp. 1910-1922, 2008.

[7] H. Xu, X. Wu, H. R. Sadjadpour, and J. Garcia-Luna-Aceves, "A unified analysis of routing protocols in MANETs," IEEE Trans. on Communications, vol. 58, no. 3, pp. 911-922, 2010.

[8] M. Grossglauser and D. N. C. Tse, "Mobility increases the capacity of ad hoc wireless networks," IEEE/ACM Trans. on Networking, vol. 10, no. 4, pp. 477-486, 2002.

[9] Q. Wang, X. Wang, and X. Lin, "Mobility increases the connectivity of k-hop clustered wireless networks," in Proc. ACM MobiCom 2009.

[10] M. Franceschetti, O. Dousse, D. N. C. Tse, and P. Thiran, "Closing the gap in the capacity of wireless networks via percolation theory," IEEE Trans. on Information Theory, vol. 53, no. 3, pp. 1009-1018, 2007.

[11] M. Neely and E. Modiano, "Capacity and delay tradeoffs for ad hoc mobile networks," IEEE Trans. on Information Theory, vol. 51, no. 6, pp. 1917-1937, 2005.

[12] S. Toumpis and A. J. Goldsmith, "Large wireless networks under fading, mobility, and delay constraints," in Proc. IEEE INFOCOM 2004.

[13] C. Hu, X. Wang, and F. Wu, "Motioncast: On the capacity and delay tradeoffs," in Proc. ACM MobiHoc 2009.

[14] A. E. Gamal, J. Mammen, B. Prabhakar, and D. Shah, "Throughputdelay trade-off in wireless networks," in Proc. IEEE INFOCOM 2004.

[15] G. Sharma and R. Mazumdar, "Scaling laws for capacity and delay in wireless ad hoc networks with random mobility," in Proc. IEEE ICC 2004.

[16] X. Lin, G. Sharma, R. Mazumdar, and N. Shroff, "Degenerate delay-capacity tradeoffs in ad-hoc networks with brownian mobility," IEEE/ACM Trans. on Networking, vol. 14, no. SI, p. 2784, 2006.

[17] G. Sharma, R. Mazumdar, and N. Shroff, "Delay and capacity trade-offs in mobile ad hoc networks: A global perspective," IEEE/ACM Trans. on Networking, vol. 15, no. 5, pp. 981-992, 2007.

[18] X. Wang, Q. Peng, and Y. Li, "Cooperation achieves optimal multicast capacity-delay scaling in MANET," IEEE Trans. on Communications, vol. 60, no. 10, pp. 3023-3031, 2012.

[19] C. Wang, C. Jiang, X.-Y. Li, S. Tang, Y. He, X. Mao, and Y. Liu, "Scaling laws of multicast capacity for power-constrained wireless networks under Gaussian channel model," IEEE Trans. on Computers, vol. 61, no. 5, pp. 713-725, 2012.

[20] A. Agarwal and P. R. Kumar, "Capacity bounds for ad hoc and hybrid wireless networks," ACM SIGCOMM Computer Communication Review, vol. 34, no. 3, pp. 71-81, 2004.

[21] S. Toumpis and A. J. Goldsmith, "Capacity regions for wireless ad hoc networks," IEEE Trans. on Wireless Communications, vol. 2, no. 4, pp. 736-748, 2003.

[22] T. Cover and J. Thomas, Elements of information theory. John Wiley and sons, 2006.

[23] Y. Wang, X. Chu, X. Wang, and Y. Cheng, "Optimal Multicast Capacity and Delay Tradeoffs in MANETs: A Global Perspective," in Proc. IEEE INFOCOM 2011.

[24] A. El Gamal, J. Mammen, B. Prabhakar, and D. Shah, "Optimal throughput-delay scaling in wireless networks-Part II: constant-size packets," IEEE Trans. on Information Theory, vol. 52, no. 11, pp. 51115116, 2006.

[25] L. Ying, S. Yang, and R. Srikant, "Optimal delay-throughput tradeoffs in mobile ad hoc networks," IEEE Trans. on Information Theory, vol. 54, no. 9, pp. 4119-4143, 2008.

[26] L. Ying and R. Srikant, "Optimal delay-throughput trade-offs in mobile ad-hoc networks: Hybrid random walk and one-dimensional mobility models," in Proceedings of ITA workshop, 2007.

[27] L. Ying, S. Yang, and R. Srikant, "Coding achieves the optimal delaythroughput tradeoffs in mobile ad-hoc networks," in Proc. WiOpt 2007.

[28] X. Wang, Y. Bei, Q. Peng, and L. Fu, "Speed improves delay-capacity tradeoff in MotionCast," IEEE Trans. on Parallel and Distributed Systems, vol. 22, no. 5, pp. 729-742, 2011.

[29] S. Zhou and L. Ying, "On delay constrained multicast capacity of largescale mobile ad-hoc networks," in Proc. IEEE INFOCOM 2010.

[30] U. Lee, K. Lee, S. Oh, and M. Gerla, "Understanding the capacity and delay scaling laws of delay tolerant networks: A unified approach," UCLA Technical Report: TR, Tech. Rep., 2008.

[31] M. Garetto and E. Leonardi, "Restricted Mobility Improves DelayThroughput Tradeoffs in Mobile Ad Hoc Networks," IEEE Trans. on Information Theory, vol. 56, no. 10, pp. 5016-5029, 2010.

[32] S. Li, Y. Liu, and X.-Y. Li, "Capacity of large scale wireless networks under gaussian channel model," in Proc. ACM MobiCom 2008.

[33] X. Wang, W. Huang, S. Wang, J. Zhang, and C. Hu, "Delay and capacity tradeoff analysis for motioncast," IEEE/ACM Trans. on Networking, vol. 19, no. 5, pp. 1354-1367, oct. 2011.

[34] X.-Y. Li, "Multicast capacity of wireless ad hoc networks," IEEE/ACM Trans. on Networking, vol. 17, no. 3, pp. 950-961, 2009.

[35] V. Vapnik and A. Chervonenkis, "On the uniform convergence of relative frequencies of events to their probabilities," Theory of Probability and its Applications, vol. 16, no. 2, pp. 264-280, 1971.

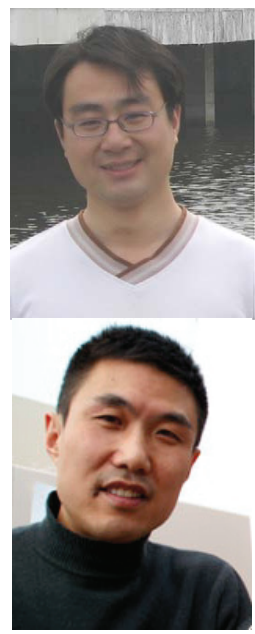

Cheng Wang received his $\mathrm{PhD}$ degree in $\mathrm{De}$ partment of Computer Science at Tongji University in 2011. Currently, he is a research professor of Computer Science at Tongji University. His research interests include wireless networking, mobile social networks, and cloud computing. from University of Illinois at Urbana-Champaign, a Bachelor degree at Department of Computer Science and a Bachelor degree at Department of Business Management from Tsinghua University, P.R. China, both in 1995. His research interests include mobile computing, cyber physical systems, wireless networks, security and privacy, and algorithms. He is an editor of several journals, including IEEE Transaction on Parallel and Distributed Systems, IEEE Transaction on Mobile Computing.

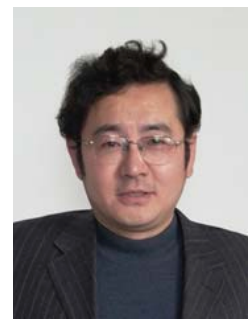

Changjun Jiang received the Ph.D. degree from the Institute of Automation, Chinese Academy of Sciences, Beijing, China, in 1995. Currently he is a Professor with the Department of Computer Science and Engineering, Tongji University, Shanghai. His current areas of research are concurrent theory, Petri net, and formal verification of software, wireless networks, concurrency processing and intelligent transportation systems.

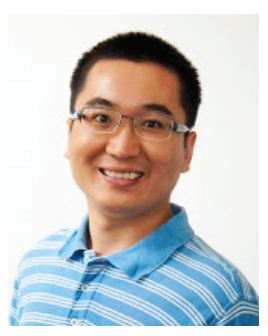

Huiya Yan received his Ph.D. in Mathematics from West Virginia University in 2009. Currently he is an Associate Professor of Mathematics at University of Wisconsin-La Crosse. His research is in graph theory and its applications. 